\title{
Involvement of PARP1 in the regulation of alternative splicing
}

Elena Matveeva ${ }^{1}$, John Maiorano ${ }^{1}$, Qingyang Zhang ${ }^{2}$, Abdallah M Eteleeb ${ }^{3}$, Paolo Convertini ${ }^{1,4}$, Jing Chen ${ }^{1}$, Vittoria Infantino ${ }^{4}$, Stefan Stamm ${ }^{1}$, Jiping Wang ${ }^{2}$, Eric C Rouchka ${ }^{3}$, Yvonne N Fondufe-Mittendorf ${ }^{1}$

${ }^{1}$ Department of Molecular and Cellular Biochemistry, University of Kentucky, Lexington, KY, USA; ${ }^{2}$ Department of Molecular Biosciences, Northwestern University, Evanston, IL, USA; ${ }^{3}$ Department of Computer Engineering and Computer Science, University of Louisville, Louisville, KY, USA; ${ }^{4}$ Department of Science, University of Basilicata, Potenza, Italy

Specialized chromatin structures such as nucleosomes with specific histone modifications decorate exons in eukaryotic genomes, suggesting a functional connection between chromatin organization and the regulation of pre-mRNA splicing. Through profiling the functional location of Poly (ADP) ribose polymerase, we observed that it is associated with the nucleosomes at exon/intron boundaries of specific genes, suggestive of a role for this enzyme in alternative splicing. Poly (ADP) ribose polymerase has previously been implicated in the PARylation of splicing factors as well as regulation of the histone modification H3K4me3, a mark critical for co-transcriptional splicing. In light of these studies, we hypothesized that interaction of the chromatin-modifying factor, Poly (ADP) ribose polymerase with nucleosomal structures at exon-intron boundaries, might regulate pre-mRNA splicing. Using genome-wide approaches validated by gene-specific assays, we show that depletion of PARP1 or inhibition of its PARylation activity results in changes in alternative splicing of a specific subset of genes. Furthermore, we observed that PARP1 bound to RNA, splicing factors and chromatin, suggesting that Poly (ADP) ribose polymerase serves as a gene regulatory hub to facilitate co-transcriptional splicing. These studies add another function to the multi-functional protein, Poly (ADP) ribose polymerase, and provide a platform for further investigation of this protein's function in organizing chromatin during gene regulatory processes.

Keywords: Epigenetics; cotranscriptional splicing; gene regulation; chromatin; PARP1

Cell Discovery (2016) 2, 15046; doi:10.1038/celldisc.2015.46; published online 16 February 2016

\section{Introduction}

Alternative splicing is a cellular process that serves to markedly increase the transcriptome and protein biodiversity within eukaryotic cells [1]. In humans, $\sim 95 \%$ of multi-exonic genes are alternatively spliced $[2,3]$. Alternative splicing events (ASEs) direct tissue-, cell type- and developmental stage-specific gene expression patterns in eukaryotes [4]. Alternative splicing decisions have important roles in many cellular processes, ranging from sex determination in fruit flies to programmed cell death in human cells, and are implicated in human disease $[6,5]$.

Correspondence: Yvonne N Fondufe-Mittendorf

Tel: +859 323 5549; Fax: +859 257 2283;

E-mail: y.fondufe-mittendorf@uky.edu

Received 21 October 2015; accepted 11 November 2015
Alternative splicing is regulated by the binding of trans-acting factors to their target sites on pre-mRNA. These trans-acting factors promote or reduce the usage of a particular splice site. Emerging data suggest that the information present at these splice sites and the binding of these factors in vivo may not be sufficient to define exons or regulate alternative splicing [7]. This has led to the 'co-transcriptional splicing hypothesis' [8], which suggests that splicing and transcription occur at the same time, with local chromatin structure being responsible for the cross-talk between transcription and splicing. Building on this idea, several studies showed that nucleosomes and/or specific histone modifications affect both the association of splicing factors (SFs) with chromatin and the efficiency of the splicing process [8-10].

The nucleosome, the basic repeating unit of chromatin, consists of $147 \mathrm{bp}$ of DNA wrapped around a histone octamer; two copies each of histone H2A, H2B, 
H3 and H4. The location of nucleosomes on the eukaryotic genome regulates cellular processes that require DNA to transcribe, replicate, recombine and repair DNA. Although the roles of nucleosomes positioned at promoters have been widely studied in transcriptional regulation, the roles of nucleosomes in splicing regulation are less well understood [11, 12]. The positioning of nucleosomes at exons $[13,14]$ is dependent on several factors including the intrinsic DNA sequence [15, 16], DNA methylation levels [17, 18] and histone modifications [19]. Indeed, nucleosomes regulate RNA polymerase elongation kinetics, thus aiding in the recognition of weak splice sites $[7,17]$. These nucleosomes typically associate with DNA that has a high GC content, high DNA methylation pattern and specific histone post-translational modifications (PTMs), which are all factors that influence nucleosome stability [7, 17, 20-23]. In support of a splicing regulatory role of histone PTMs, data in yeast show elevated transcription levels are associated with reduced histone occupancy. In addition, the transcription-associated H3K36me3 modification is reduced at alternatively spliced exons compared with constitutive exons [22, 24].

As alternative splicing appears to occur cotranscriptionally in vivo, we hypothesize that factors affecting chromatin stability and dynamics regulate alternative splicing. Although the roles of histone PTMs and DNA methylation in co-transcriptional splicing have been extensively studied, the role of other chromatin modulators in this process is less studied. One potential modulator is poly (ADP)-ribose polymerase 1 (PARP1), which is known to remodel chromatin through PARylation (addition of poly (ADP) ribose moieties) of histones to regulate transcription. Through profiling of PARP1 chromatinbinding sites, we found sharp occupancy peaks for PARP1 at internal intron/exon boundaries, suggesting a role in pre-mRNA splicing. Our studies suggest that PARP1 acts as a structural chromatin entity and an adapter molecule bridging chromatin and RNA, and as a recruiter of SFs. These studies comprise the first comprehensive genome-wide determination of PARP1 in mediating gene regulation at the splicing level.

\section{Results}

We assessed the genomic distribution of PARP1 in S2 Drosophila cells by nucleosome-chromatin immunoprecipitation using PARP1 antibody followed by deep sequencing (nuc-ChIP-seq) (Supplementary Figure S1). The Drosophila system provides a convenient model to test the effect of PARP1 on gene regulation as Drosophila contains only one PARP1 gene and a tankyrase, compared with at least 18 different PARP genes in humans $[25,26]$.

\section{PARP1 preferentially binds active promoters}

Previous studies using ChIP-chip experiments as well our recent nuc-ChIP-seq show that PARP1 binds
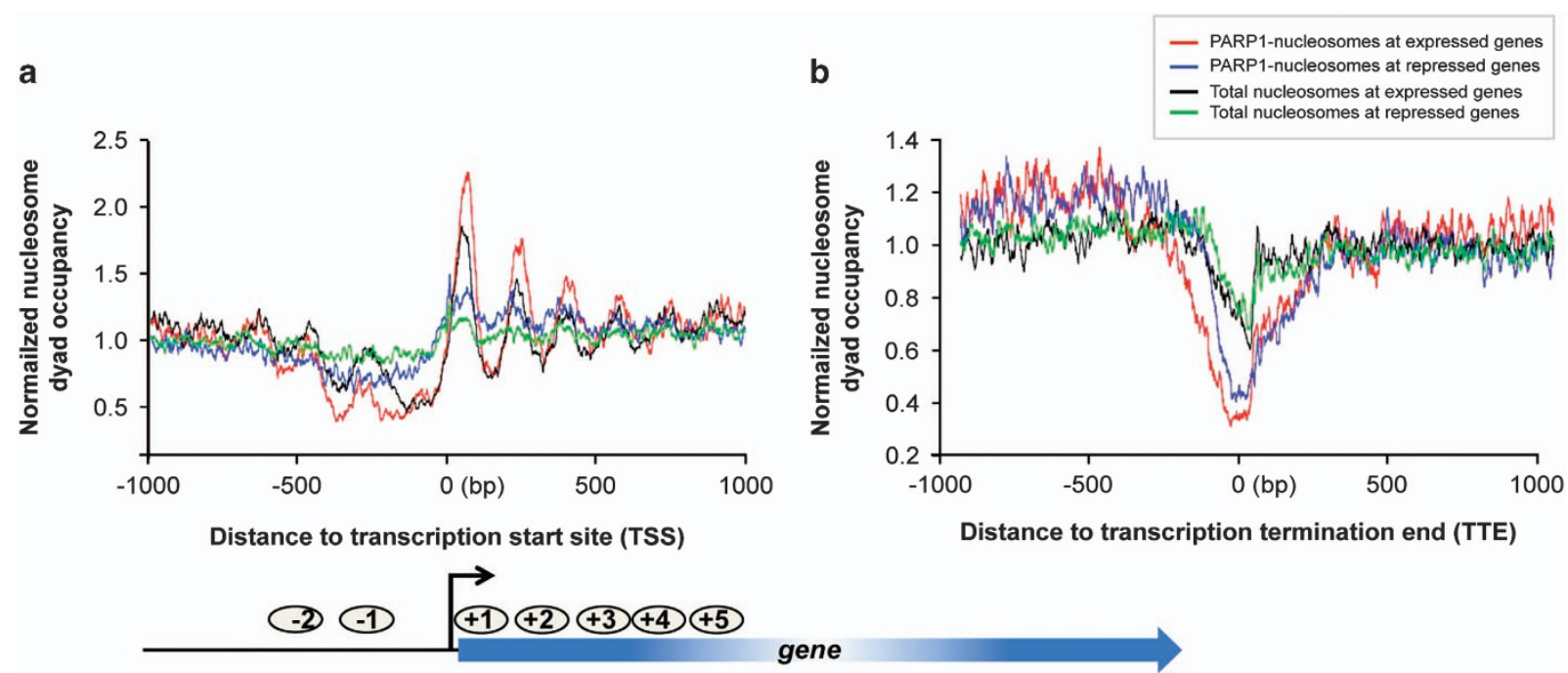

Figure 1 PARP1 is enriched around the promoters of active genes and depleted at the ends of genes. Dyad density plot of PARP1-bound nucleosomes and total nucleosomes in S2 cells around (a) transcription start sites (TSSs) and (b) transcription termination ends (TTEs). To control for the difference in the total number of tags, dyad density scores are normalized by the average density over the genome. 
to active promoter regions in human cells [27, 28]. We sought to determine whether this is true in the Drosophila genome, where the presence of a single gene permits a higher resolution nuc-ChIP-seq analysis. Using this analysis, we examined the distribution of PARP1-nucleosome reads within $2 \mathrm{~kb}$ upstream and downstream of annotated transcription start sites (TSSs), as described in the Materials and Methods section. We observed that PARP1 associates with the +1 and +2 nucleosomes of active promoters (Figure 1a) and not with the nucleosomes at the transcription termination ends (TTEs, Figure 1b). These data are consistent with previous lower resolution studies that show PARP1 enriched at +1 and +2 nucleosomes of heat-shock genes [29, 30] as well as our recent high-resolution analyses of PARP1 binding in human cells [28]. Based on this observation, we further quantified the relationship between gene expression and PARP1 interaction with promoters, by calculating the Pearson correlation between gene expression and PARP1-nuc-ChIP-seq read depth across -50 to +500 bp surrounding annotated promoter regions. PARP1 association correlates positively with gene expression (Pearson correlation $R=0.427 ; P=2.2 \mathrm{e}-16$ ). Performing the same analyses at the ends of genes showed a very weak/no correlation with gene expression levels $(R=0.0196 ; P=0.025)$.

\section{PARPI associates with exonic nucleosomes}

While profiling PARP1 genomic location, we observed PARP1-nucleosome signals at exon-intron boundaries. To test whether the binding of PARP1 in coding regions was related to the chromatin architecture around exon/intron junctions in Drosophila, a fine chromatin structure map of PARP1 occupancy across the coding regions of active genes and silent genes was generated (Figure 2). These analyses showed that PARP1 associated with nucleosomes within exons, irrespective of the transcriptional status of the genes (Pearson correlation $R=0.325 ; \quad P=1.5 \mathrm{e}-16$ ) (Figure $2 \mathrm{a}-\mathrm{d}$ ). We next addressed whether the sharp peak of PARP1-bound nucleosomes observed at the exon/intron and intron/exon boundaries was a special feature of PARP1 chromatin structure at these regions. We compared the profiles of total nucleosome occupancy with those of PARP1-bound nucleosomes at the intron-exon boundaries (Figure $2 \mathrm{a}$ and c) and exon-intron boundaries (Figure $2 \mathrm{~b}$ and $\mathrm{d}$ ). We observed a difference in both profiles: higher peak in the PARP1-bound nucleosome profile with a deeper valley past these peaks than with total nucleosome profiles. From these analyses, we concluded the observed peaks in the PARP1-nuc-seq data are not solely the consequence of increased nucleosome occupancy in this region, but an enrichment of PARP1-bound nucleosomes at these regions.

To differentiate the observed effect of PARP1 at TSSs with those at the internal exons, we asked if there was a difference between PARP1-bound nucleosomes at the first exon/intron boundaries compared with PARP1-bound nucleosomes at internal exon/intron boundaries. To address this question, PARP1-bound nucleosome footprints centered on the 5 ' sites of all exons were constructed. This analysis showed low levels of PARP1-bound nucleosomes at the ends of first exons (excluding $\pm 250 \mathrm{bp}$ surrounding annotated TSSs) (Figure 3a), whereas PARP1-bound nucleosomes were abundantly positioned at the start of internal exons (Figure 3b). To ensure that nuc-ChIP seq technique did indeed pull down PARP1 targets, chromatin was digested with different amounts of MNase, resulting in varying lengths of chromatin (Supplementary Figure S2A). ChIP-qPCR (quantitative real-time polymerase chain reaction) was performed to validate PARP1 binding at target and non-target genes (Supplementary Figure S2B-D). The results show that PARP1-nucleosome binding is specific validating the nuc-ChIP-seq results (PARP1nuc-ChIP profiles are shown in Supplementary Figure S2E). Analyses of gene ontology by the Database for Annotation, Visualization and Integrated Discovery (DAVID) Bioinformatics Resources (version 6.7) [31] of the genes bound at the internal exon/ intron boundaries by PARP1 showed that these genes were involved in several important gene regulatory processes (Supplementary Figure S2F and Supplementary Table S1).

\section{PARP1 associates with GC-rich nucleosomes and with active histone modifications}

Previous work demonstrates that the preference for nucleosome positioning over exons is influenced by nucleotide sequence composition. Across eukaryotes, exon sequences tend to have elevated GC content compared with flanking introns [7, 17, 20, 32]. As higher GC content confers stability to exonic nucleosomes [15, 33], we sought to determine whether PARP1-bound nucleosomes are particularly GC-rich. We found that as the $\mathrm{GC}$ percentile increases, more PARP1 reads (compared with total nucleosomes) were mapped, indicating that PARP1-associated nucleosomes at the internal exon/intron boundaries are, on average, GC-rich (Figure 3c). GC-rich nucleosomes have been suggested to bind to weak splice sites, 

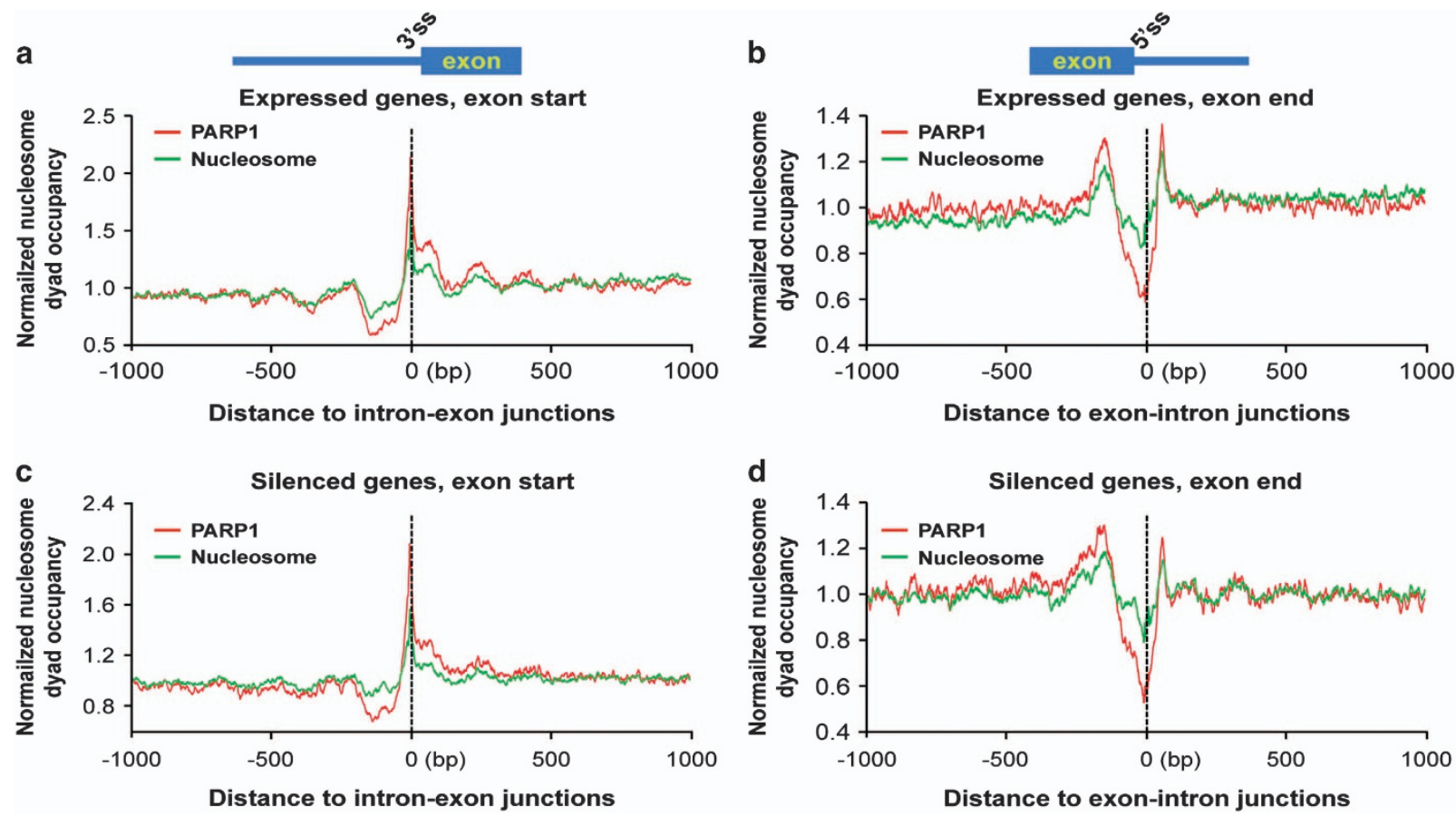

Figure 2 PARP1 demarcates exons and is enriched at intron/exon and exon/intron boundaries irrespective of the transcriptional state of the genes. For this calculation, intron-containing genes were used and curves were normalized by genome-wide average. Dyad density of PARP1 nucleosomes at (a) start of exons (intron/exon) and (b) end of exons (exon/intron) of active genes, respectively. Dyad density of PARP1 nucleosomes at (c) start of exons (intron/exon) and (d) end of exons (exon/intron) of inactive or silent genes, respectively. To control for the effect of nucleosomes, dyad density scores are normalized by the average density over the genome (see Supplementary Figure S1).

slowing down the rate of RNAPII elongation [20, 34], which might promote co-transcriptional splicing.

We next investigated the association of PARP1 with specific histone modifications. We downloaded a large set of histone modification data on S2 Drosophila cell line from the modENCODE project [35]. Analyses of our PARP1-nuc-ChIP-seq results (PARP1 binding) showed an overlap of PARP1binding with several active histone PTMs ChIP-seq data (Figure 3d) such as H3K4me3, H3K36me3, H4K16ac but not with the repressive histone PTM H3K27me3 nor H1.

\section{Global ASEs mediated by PARPI and its PARylation} activity

As PARP1 is associated with nucleosomes at exon boundaries, we hypothesized that PARP1-associated chromatin structure might function in alternative splicing. To test the functionality of PARP1 in alternative splicing, we used small interfering (siRNA) to knockdown PARP1 in S2 Drosophila cells (compared with cells treated with LacZ control siRNA). PARP1-knockdown efficiency was determined at both protein and mRNA levels (Supplementary
Figure S3A and B). As previous studies have shown that PARylation of proteins within the spliceosomal complex is important for their activity [36, 37], we also asked whether the observed effects on alternative splicing were dependent on PARP1's PARylation activity by inhibiting PARylation using PJ-34 [29, 38] (Supplementary Figure S3C).

To address the global impact of PARP1 on alternative splicing, we isolated total RNA in two biological replicates from control (non-treated), PARP1 siRNA- and PJ-34-treated cells. Sequencing of these RNAs on an Illumina HiSeq 2500 yielded $>56$ million 100-bp paired-end RNA-seq reads. First, we aligned reads to the entire gene body of PARP1 and confirmed a reduction in PARP1 expression of $\sim 30 \%\left(P<10^{-3}\right)$. This result is consistent with the quantitative reverse-transcription PCR (qRT-PCR) data (Supplementary Figure S3B), and confirms PARP1 depletion after PARP1 siRNA treatment (Supplementary Table S2). We next used these RNAseq data sets (control, PARP1 KD and PARylation inhibited) to assess whether these treatments resulted in changes in (i) gene expression and (ii) alternative splicing. 
a
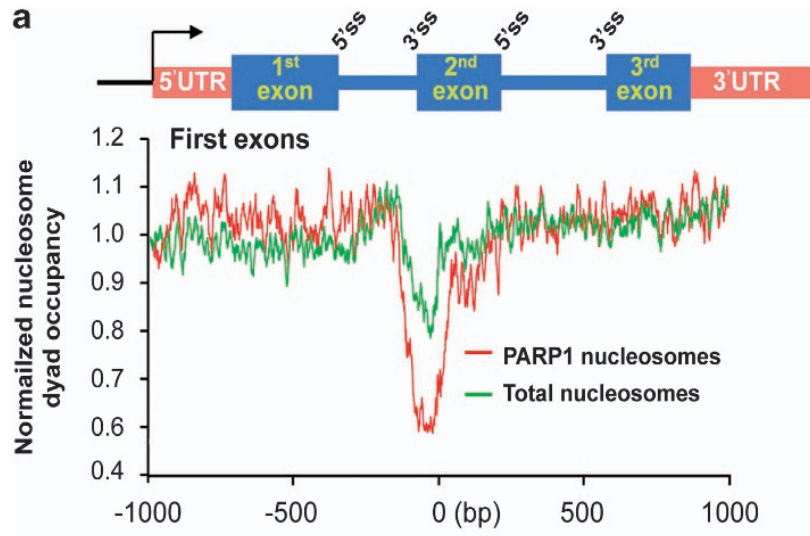

Distance to intron-exon junctions

b

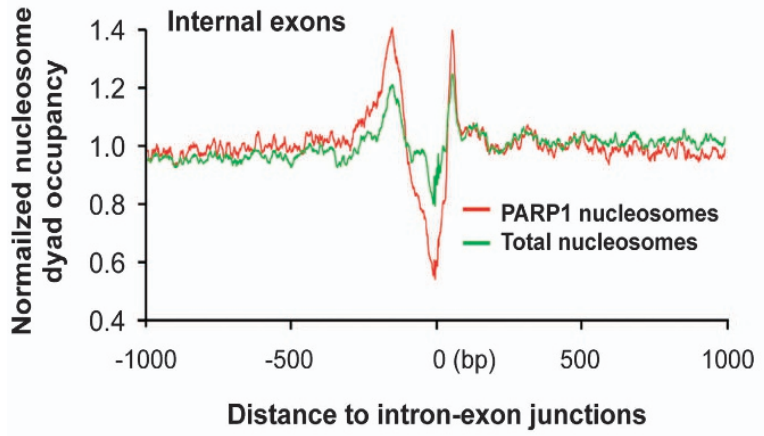

C
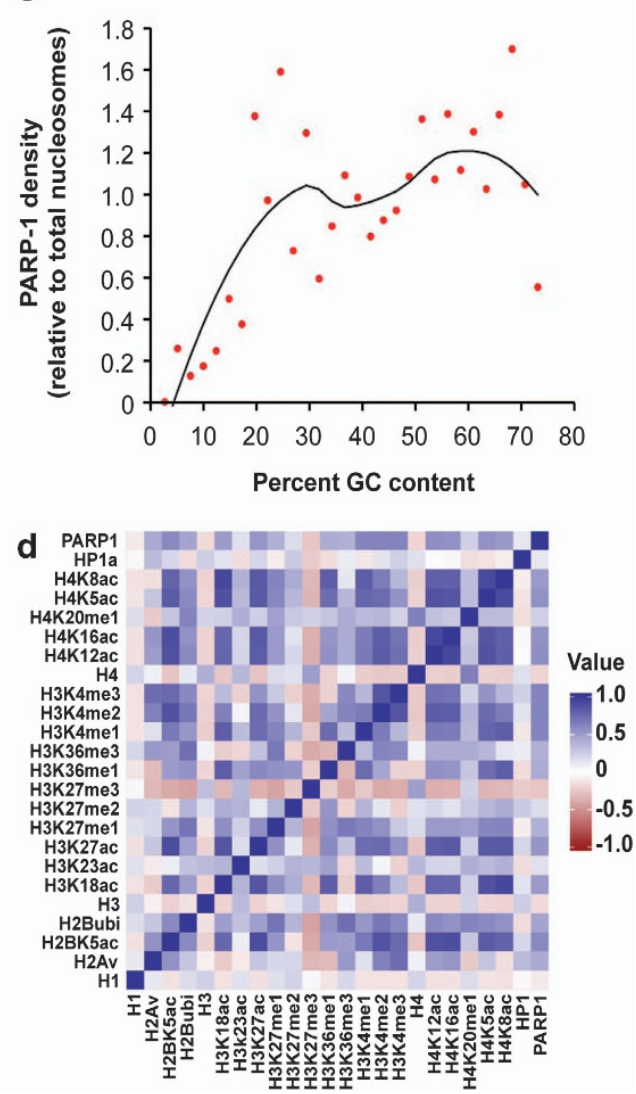

Figure 3 Characteristic features of PARP1-bound nucleosomes. (a) PARP1-bound nucleosomes (red curve) are not enriched at first exons boundaries but are (b) highly enriched at internal exons (tag densities were measured at $\pm 1000 \mathrm{bp}$ ), even if the effect of total nucleosome (green curve) is subtracted. For this calculation only intron-containing genes were used. (c) PARP1 associates with GC-rich nucleosomes. Plot shows PARP1-bound nucleosome density relative to total nucleosome density as a function of GC content. The black curve is the fitted smoothing line by loess method (local regression) using locally weighted polynomial regression analyses. (d) Heatmap showing that PARP1 binding overlaps with chromatin regions occupied by specific histone modifications. Pearson correlations of PARP1 and the following epigenetic marks (all with $P$-values $<10^{10}$ ).

\begin{tabular}{ll}
\hline Epigenetic Marker & P-value \\
\hline H1 & -0.100084 \\
H2Av & 0.429887 \\
H2BK5ac & 0.586200 \\
H2Bubi & 0.454395 \\
H3K4me1 & 0.611728 \\
H3K4me2 & 0.621749 \\
H3K4me3 & 0.611728 \\
H3K18ac & 0.503160 \\
H3K23ac & 0.146093 \\
H3K27ac & 0.525930 \\
H3K27me1 & 0.387621 \\
H3K27me2 & 0.174618 \\
H3K27me3 & -0.257703 \\
H3K36me1 & 0.411887 \\
H3K36me3 & 0.390735 \\
H4K5ac & 0.543766 \\
H4K8ac & 0.508607 \\
H4K12ac & 0.564942 \\
H4K16ac & 0.553435 \\
H4K20me1 & 0.238543 \\
HP1a & 0.121145 \\
\hline
\end{tabular}


Gene expression patterns mediated by PARPI and PARylation

Using the Tuxedo protocol for RNA-seq analysis [39], PARP1 depletion resulted in $\sim 849$ differentially expressed genes (DEGs) $(P \leqslant 0.05)$, of which 210 were upregulated (Supplementary Table S2C) and 639 were downregulated (Supplementary Table S2D). We used categoryCompare [40] to determine the function of these DEGs (Supplementary Figure S4A). Genes upregulated after PARP1 knockdown are mainly involved in nucleotide metabolism, ATP synthesis and spindle (centromere) formation, whereas those downregulated genes are involved in differentiation, growth, migration and development (Supplementary Table S2E). Inhibition of PARylation affected the expression of 1281 genes; 974 of these were upregulated (Supplementary Table S2A) and 307 were downregulated (Supplementary Table S2B) $(P \leqslant 0.05)$. Interestingly, the top category of genes upregulated after PARylation inhibition are involved in the spliceosome and in RNA polymerase-processing pathways, whereas downregulated genes are involved in neuronal differentiation, cell death and immune response (Supplementary Table S2F). Comparison of the DEGs mediated by PARP1 knockdown and PARylation inhibition reveals 182 genes common to both (Supplementary Figure S4A and B) with no common directionality in the gene expression profiles. Changes in gene expression were validated using qRT-PCR, with 8 out of 10 genes $(90 \%)$ in accordance with RNA-seq data (Supplementary Figure S4C).

\section{Alternative splicing patterns mediated by PARPI and PARylation}

We asked next if PARP1 or PARylation controls the expression patterns of alternative transcript isoforms at the transcriptome level. MATS (multivariate analysis of transcript splicing) [41] and MISO (mixture of isoforms) [42] were used to estimate expression levels of different mRNA isoforms (Supplementary Figure S5A). Both methods use a Bayesian approach to calculate the ASEs between two samples; however, MATS measures ASEs, whereas MISO determines both isoform and event-inclusion level [43]. We used both methods to identify targets for which PARP1 or PARylation modulates the following pre-mRNA processing event subclasses: (i) mutually exclusive exons, (ii) cassette exons (skipped exons (SEs) according to MATs analyses), (iii) alternative $3^{\prime}$ and $5^{\prime}$ splice sites and (iv) retained introns (Supplementary Figure S5A). Some events were commonly detected only by MISO (Supplementary Table S3), whereas others were detected by both methods (Figure $4 \mathrm{a}$ and $\mathrm{d}$ and Supplementary Figure S5A; Supplementary Table S4), indicating the validity of these events. MISO $(\Delta \Psi$ of 0.2 and Bayes factor $\geqslant 10$ ) detected a total of 1540 events (4.5\% of total MISO-detectable ASEs) regulated by PARP1 independent of its catalytic activity and 745 events ( $2 \%$ of total MISO-detectable ASEs) regulated through the PARylation activity of PARP1. Likewise, MATS at $P<0.005$, identified 135 events $(3 \%$ of total MATS-detectable ASEs) as regulated by PARP1 and 290 events (4\% of MATS-detectable ASEs) as regulated through the PARylation activity of PARP1 (Figure 4a). Examples of ASEs determined by MISO are illustrated using Sashimi plots (Figure $4 \mathrm{~b}$ and Supplementary Figure S5B). Overall, these results show that irrespective of the model used (MISO or MATS), PARP1 and PARylation have a broad role in regulating mRNA processing events in Drosophila $\mathrm{S} 2$ cells.

At last, we again performed categoryCompare analysis [40] to examine whether transcripts modified by PARP1/PARylation were enriched for particular functional categories (Figure 4c). The top category found to be affected by PARP1 depletion and inhibition of the PARylation activity of PARP1 were genes involved in RNA splicing, with many core and regulatory components (including members of the SR and hnRNP families). We conclude from these results that PARP1 and PARylation preferentially affect transcripts encoding protein products involved in RNA and protein-processing pathways (Figure 4c and Supplementary Table S3). Though, PARP1 depletion and PARylation inhibition affected the expression of several genes (Supplementary Figure S4A), 87\% of the genes regulated by PARP1 at the splicing level were not affected in their level of mRNA expression (Supplementary Table S2). Of note, depletion of PARP1 did not substantially affect the expression of SFs (Supplementary Table S2C and D), though PARylation did affect the expression of SFs (Supplementary Table S2A and B). This suggested that there is a possible difference in the molecular mechanisms affected by PARP1 from its enzymatic activity.

We also analyzed the overlap between PARP1/ PARylation RNA-seq data and PARP1-ChIP-seq data to determine the PARP1-bound genes that undergo PARP1/PARylation-mediated ASE changes. We observed an $\sim 23-33 \%$ commonality, respectively, in these data sets (Supplementary Table S4). Given the technical differences in protocols and the inherent loss of information in threshold and significance threshold calls, this number of genes is likely a conservative 
a

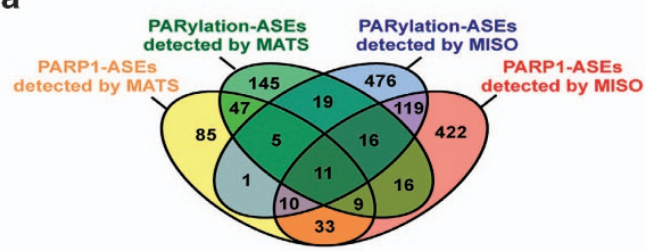

b MXE: Chr 2R:19957526 - 19960482
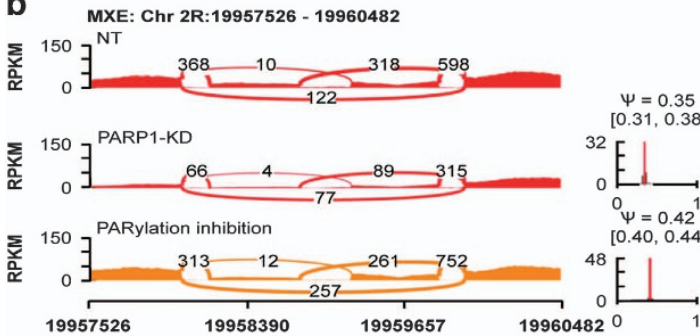

Genomic coordinate (chr 2R), + Strand

d
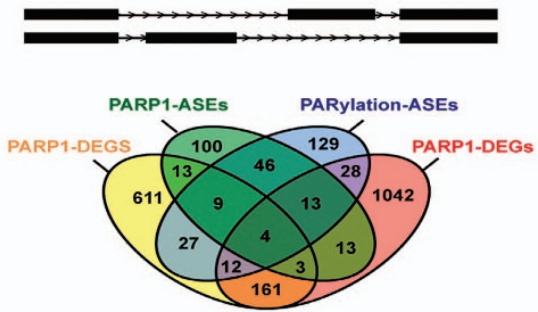

C

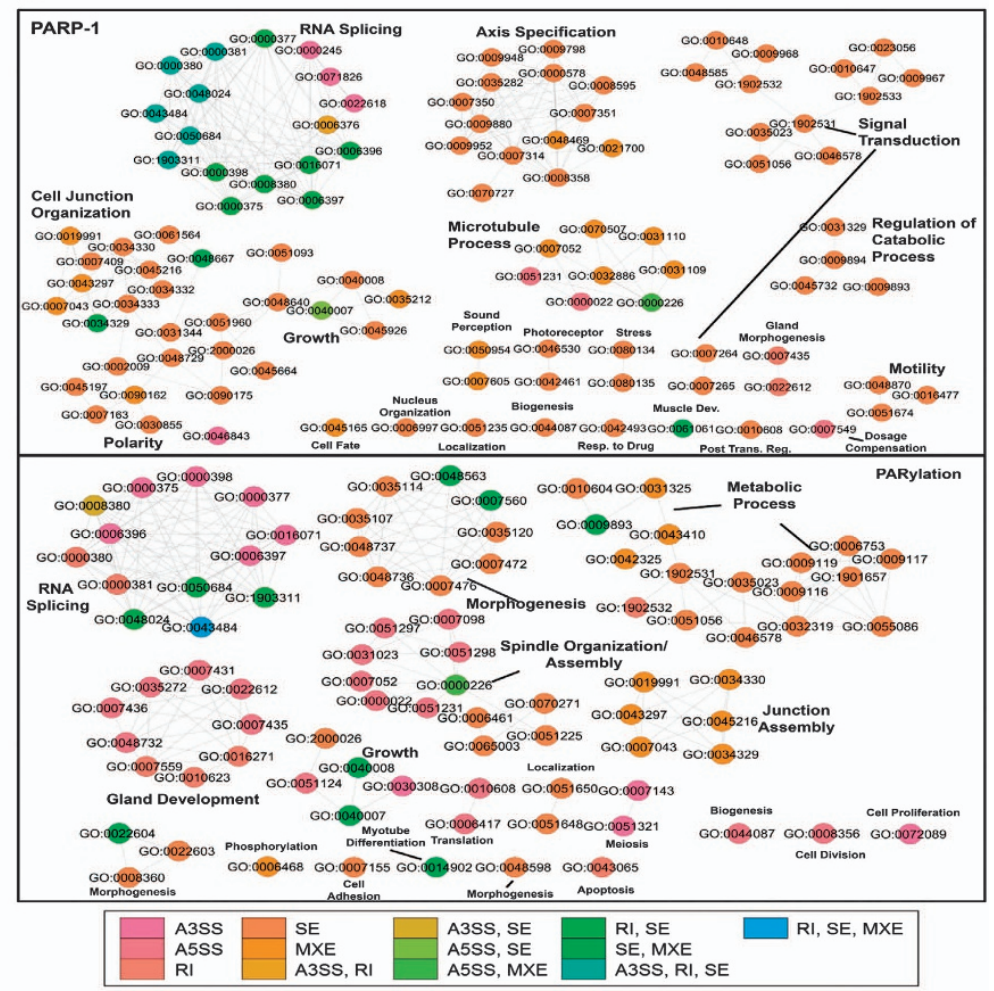

Figure 4 Global gene regulatory events mediated by PARP1 and its PARylation activity. (a) Four-way Venn diagram showing the different types of ASEs that are detected by either MATS or MISO detected by both methods. (b) Sashimi plots showing example of ASEs mediated by PARP1 and PARylation. RNA-seq read densities supporting ASEs and the estimated confidence levels are shown in the figure. (c) Visualization of the Gene Ontology Biological Process (BP) categories of PARP1 and PARylationmediated ASEs. Circles are shaded based on types of ASEs as indicated on the legend. (d) Four-way Venn diagram summarizes the number of shared proteins in each combination of the four groups. Yellow and green: PARP1-mediated DEGs and ASEs, respectively; purple and red: PARylation-mediated DEGs and ASEs, respectively $(P<0.01$, by Student's $t$-test). Numbers depicted in the intersections between circles represent the numbers of genes that are commonly regulated in two, three, or four conditions.

estimate. Next, we identified genes affected at both their expression and splicing levels by PARP1 siRNA compared with inhibition of PARylation. Surprisingly, although these analyses revealed some commonalities, the profiles were largely different (Figure 4d). For instance, PARP1 and PARylation regulated only four genes in common at both gene expression and splicing levels: CG40178, CG40191, ASCL (Acyl-CoA synthetase long-chain) and PARP1 itself.

At the gene expression level, genes shared by the PARP1 and PARylation function in several pathways, including neuron differentiation, cell death and immune response. Intriguingly, at the splicing level, PARP1 and PARylation commonly regulated 72 genes as determined by MATS, of which many are involved in splicing (Figure $4 \mathrm{a}$ and $\mathrm{c}$ ). These results are consistent with two possible modes of PARP1 function in alternative splicing that are not mutually exclusive:
(1) as a structural chromatin protein, PARP1 can directly affect alternative splicing through association with chromatin structures that affect RNA polymerase elongation and/or act as an adapter for RNA and SF binding; and (2) through PARylation of SFs PARP1 can indirectly affect splicing by regulating the activity/ expression of spliceosomal proteins (Figure 4).

\section{Validation of ASEs regulated by PARPI and PARylation}

We used semi-qRT-PCR to validate the changes in alternative splicing observed in RNA-seq analyses after PARP1 KD and PARylation inhibition. For these analyses, we chose representative genes based on the ChIP-seq data showing PARP1-binding at internal exon-intron boundaries of target genes as well as RNA-seq experiments, showing PARP1-/PARylationmediated ASEs at these genes. These splicing events fall 

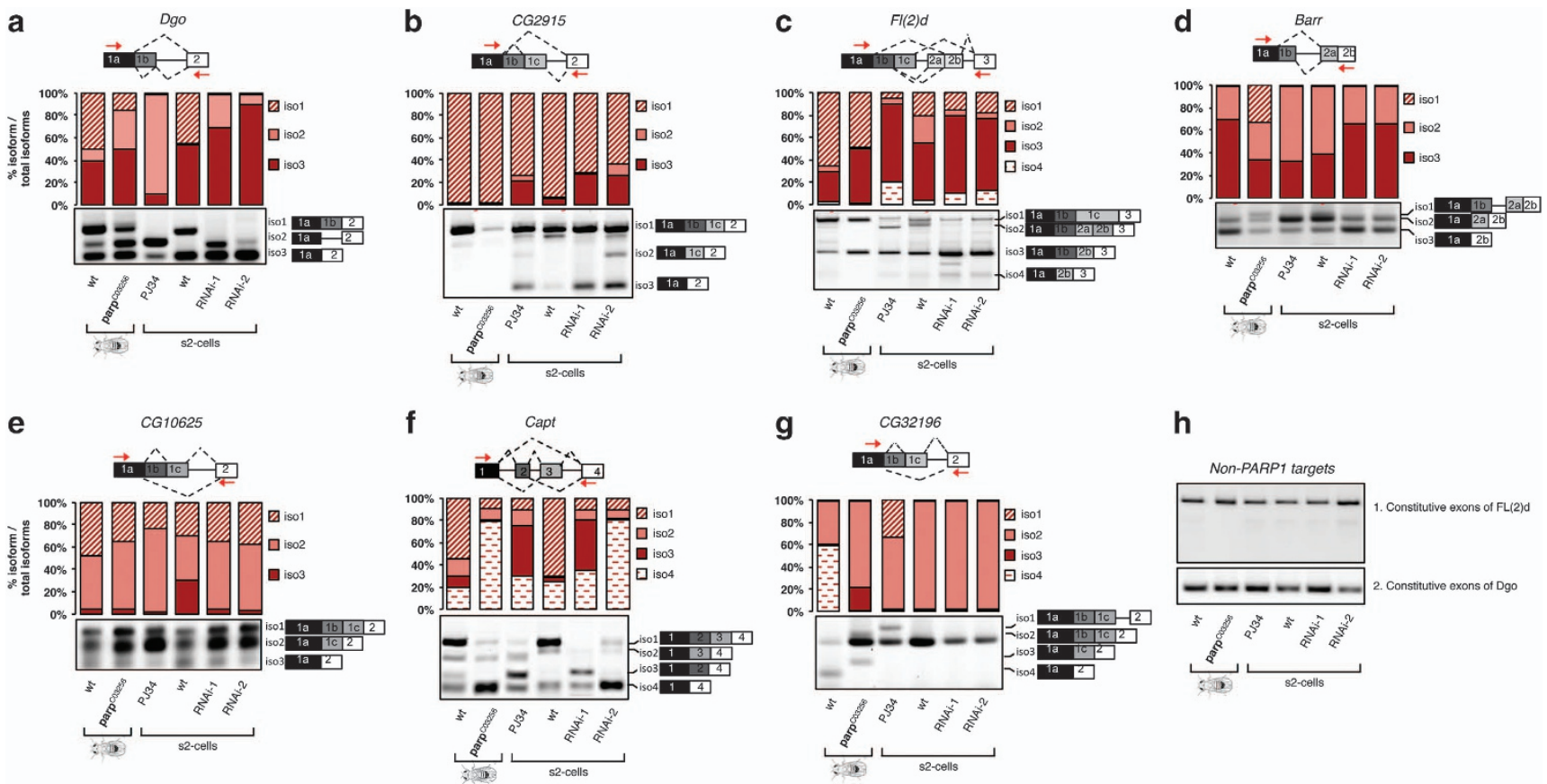

h

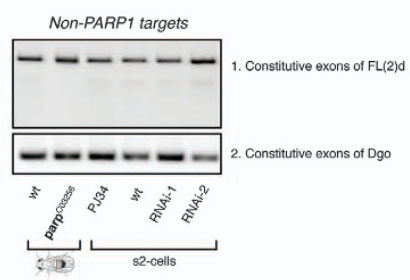

Figure 5 PARP1 regulates alternative splicing. (a-g) Measurements of ASEs at alternative exons. (h) Measured gene expression of constitutive exons. Representative gel images of the effect of PARP1 and PARylation on ASEs. Total RNA from wt and Parp ${ }^{\mathrm{C} 3256}$ flies were tested for changes in ASEs (lanes 1 and 2). S2 cells treated with PJ34 (lane 3), S2 cells treated with (i) LacZ non-targeting siRNA (lane 4), (ii) PARP1 siRNA1, (iii) PARP1 siRNA2 (KD1 and KD2, lanes 5 and 6, respectively). Bar charts represent the alternative arbitrary units from qRT-PCR measured as a rate of alternative exon included over the sum of all the alternative exons calculated from the mean intensities $(n \geqslant 3$ biological replicates, \pm S.D.; $P<0.05$ with Student's $t$-test; see Supplementary Figures S2 and 3). Black and white boxes represent constitutive exons $5^{\prime}$ and $3^{\prime}$ to the alternative exons (gray boxes), respectively. Red arrows depict locations of primers.

into several classes: (i) alternative $5^{\prime}$ splice site (SS) usage, (ii) alternative $3^{\prime}$ SS usage and (iii) exon skipping. We confirmed that knockdown of PARP1 resulted in changes in ASEs at these genes (Figure 5, lane 5: RNAi-1). A second siRNA targeting a distinct sequence within the PARP1 gene, KD2, was used to corroborate that the observed changes in alternative splicing was due to the knockdown of PARP1 and not specific to the sequence of the PARP1 siRNA (Figure 5, lane 6: RNAi-2).

We also tested and validated the effect of the PARylation activity of PARP1 in S2 cells on alternative splicing of these genes (Figure 5, Lane 3). We further confirmed the effect of PARylation on alternative splicing in a fly model, $P A R P^{C 03256}$ that expresses a short isoform of the PARP1 protein lacking the first zinc finger, with reduced PARylation activity [44]. Indeed, comparing splice isoforms between wt and PARP $P^{C 03256}$ mutant flies showed changes in ASEs (Figure 5, lane 1 and 2). The observed splicing patterns were further validated quantitatively using quantitative real-time PCR at specific alternative exons
(Supplementary Figure S6A and B). In all experimental conditions tested (PARP knockdown, PJ34-treated and $P A R P^{C 03256}$ ) compared with wild-type cells or flies, no changes in the expression at constitutive exons were observed (Figure 5 h).

\section{PARPI is recruited to chromatin and pre-mRNA}

Based on the findings above, we considered three possible recruitment mechanisms for PARP1 in splicing:

First, we asked whether the splicing effects we observed after PARP1 knockdown or PARylation inhibition were due to changes in nucleosome occupancy over these exons. For this, we carried out ChIP-qPCR analyses to measure nucleosome and PARP1 occupancy at the exons of selected genes. Knockdown of PARP1 (PARP1 KD) resulted in depletion of PARP1 occupancy at these experimental exons - Stau (Figure 6a); Fl(2)d (Figure 6b); Capt (Figure 6c) with no measurable change in nucleosome density, as measured by histone H3 occupancy (Figure 6, Lane 2). Contrary to PARP1 KD, 
a
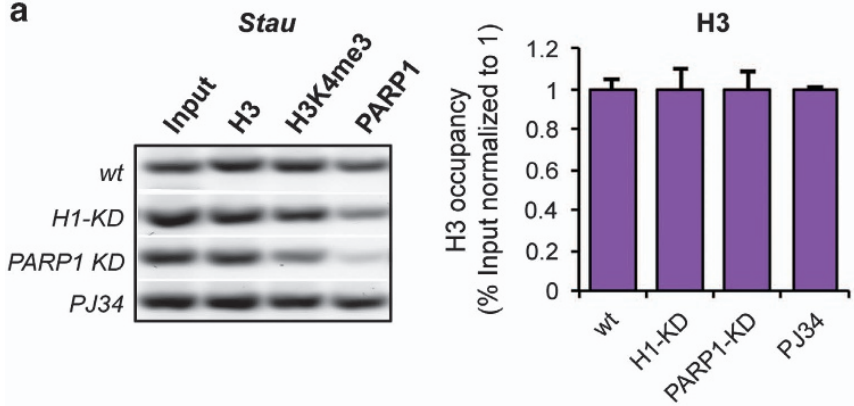

b
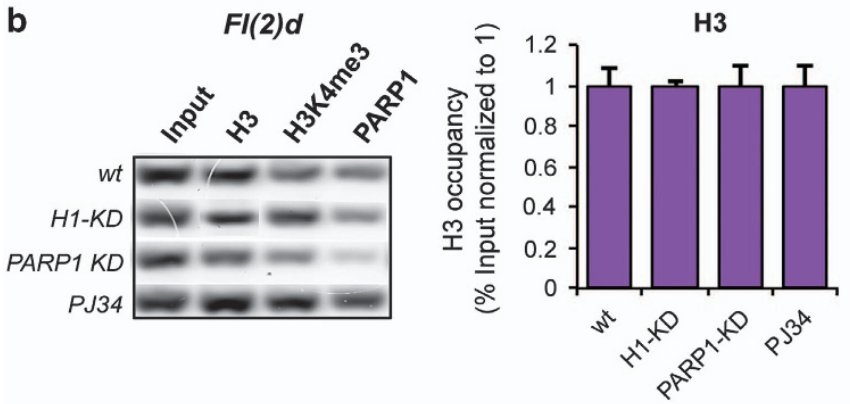

C
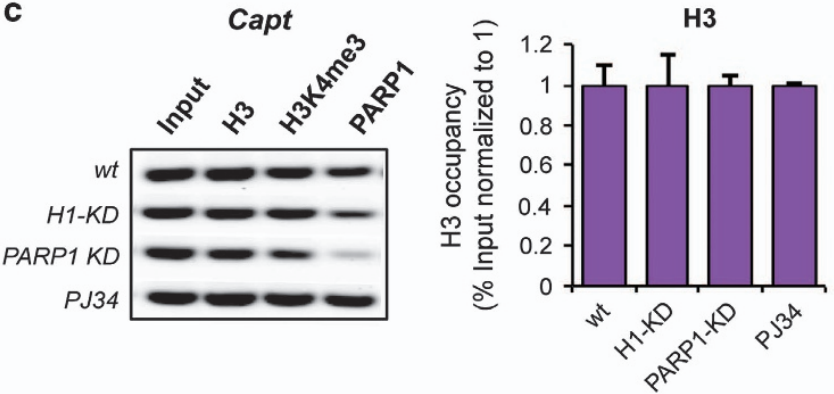
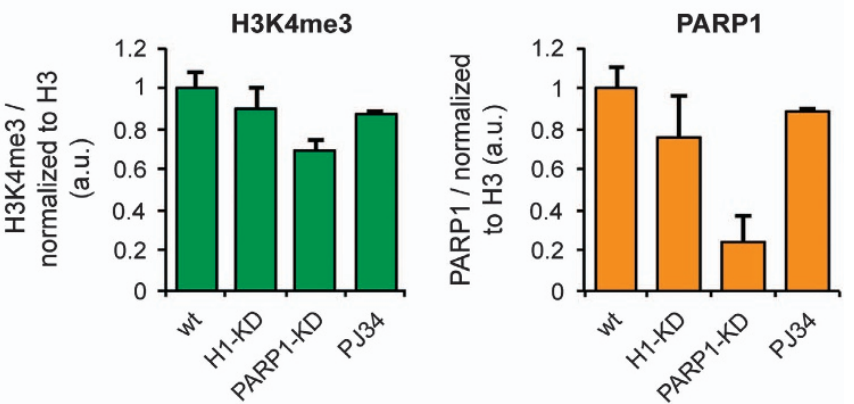

H3K4me3
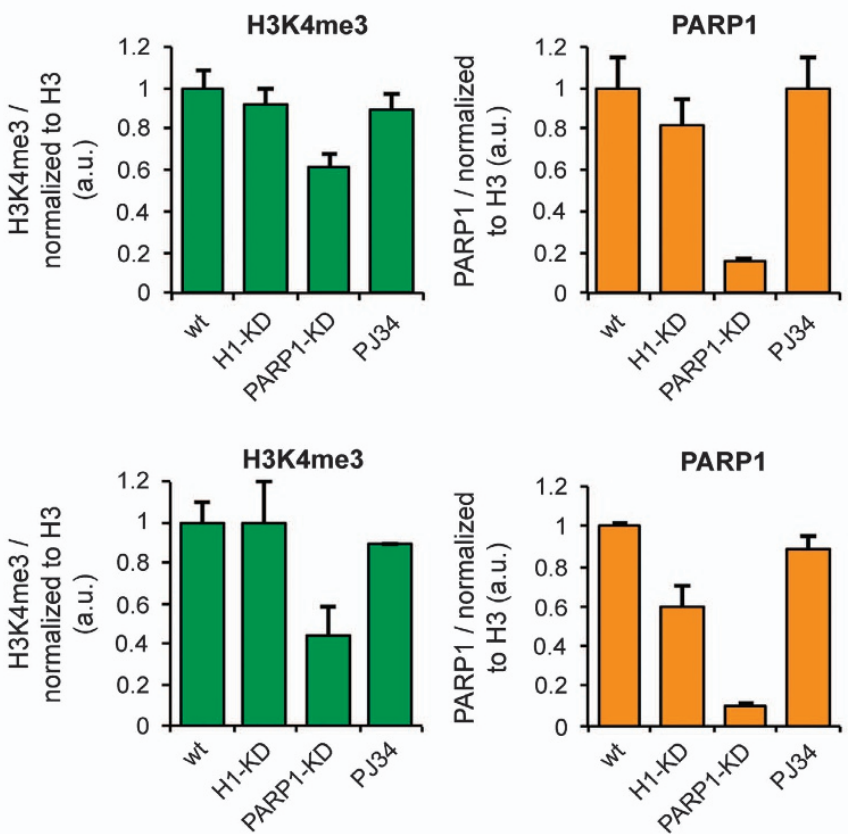

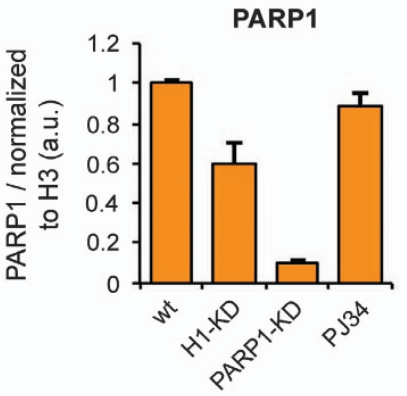

Figure 6 Co-occupancy of PARP1 and H3K4me3 at PARP1-target exons. S2 cells were transfected with siRNA targeting lacZ (NT) and PARP1 (PARP1 KD). ChIP-qPCR analysis of PARP1, nucleosome density (as measured by H3) and H3K4me3 occupancies at PARP1-target exons in (a) Stau, (b) Fl(2)d and (c) Capt1 genes were analyzed in NT, PARP1 KD and PJ34-treated S2 cells. As proof of the specificity of the observed effect, these same factors were measured in Histone H1 Knockdown cells (H1 KD). Representative inverted agarose gel images of qPCR products stained with Gelstar are shown (far left). Bar graphs show ChIP-qPCR (quantitative real-time PCR) analyses normalized to lgG control of the measured occupancies. Antibodies used are indicated above each graph; results are represented as mean plus s.e.m. ( $n \geqslant 3 ;$ Student's $t$-test, $P<0.05)$. Primers that target the alternative exons as in Supplementary Figure S5B were used in ChIP PARP1, H3K4me4 co-occupancy assays.

PARylation inhibition had no effect on nucleosome density (H3 occupancy) or PARP1 occupancy (Figure 6a-c, Lane 2 and 4), corroborating previous results [45].

Second, we asked if at these exons, PARP1 associates with specific histone modifications that have been implicated in splicing. In these studies, we focused on $\mathrm{H} 3 \mathrm{~K} 4 \mathrm{me} 3$ for several reasons: (1) H3K4me3 is proposed to facilitate SF loading [10], (2) we show both in human cells [46, 47] and S2 Drosophila cells (Figure 3d) that PARP1 binding correlates positively with H3K4me3 presence (in S2 Drosophila cells with a Pearson correlation $R=0.6215 ; P<10^{-10}$ ).
ChIP-qPCR analyses confirmed the co-presence of H3K4me3 and PARP1 at the nucleosomes of these PARP1-target exons (Figure 6a-c, Lane 3 and 4). In the absence of PARP1 (PARP KD), a concomitant reduction $(\sim 60 \%)$ of $\mathrm{H} 3 \mathrm{~K} 4 \mathrm{me} 3$ occupancy at these target exons was observed (Figure 6a-c), confirming previous studies of a role of PARP1 in H3K4me3 deposition [46]. We therefore hypothesized that this reduction is specific for PARP1 knockdown as knockdown of Histone H1, a protein that competes for nucleosomal binding with PARP1 [27], had no significant effect on $\mathrm{H} 3 \mathrm{~K} 4 \mathrm{me} 3$ or PARP1 occupancies at these exonic sites (Figure 6, Lane 3 and 4). However, at 

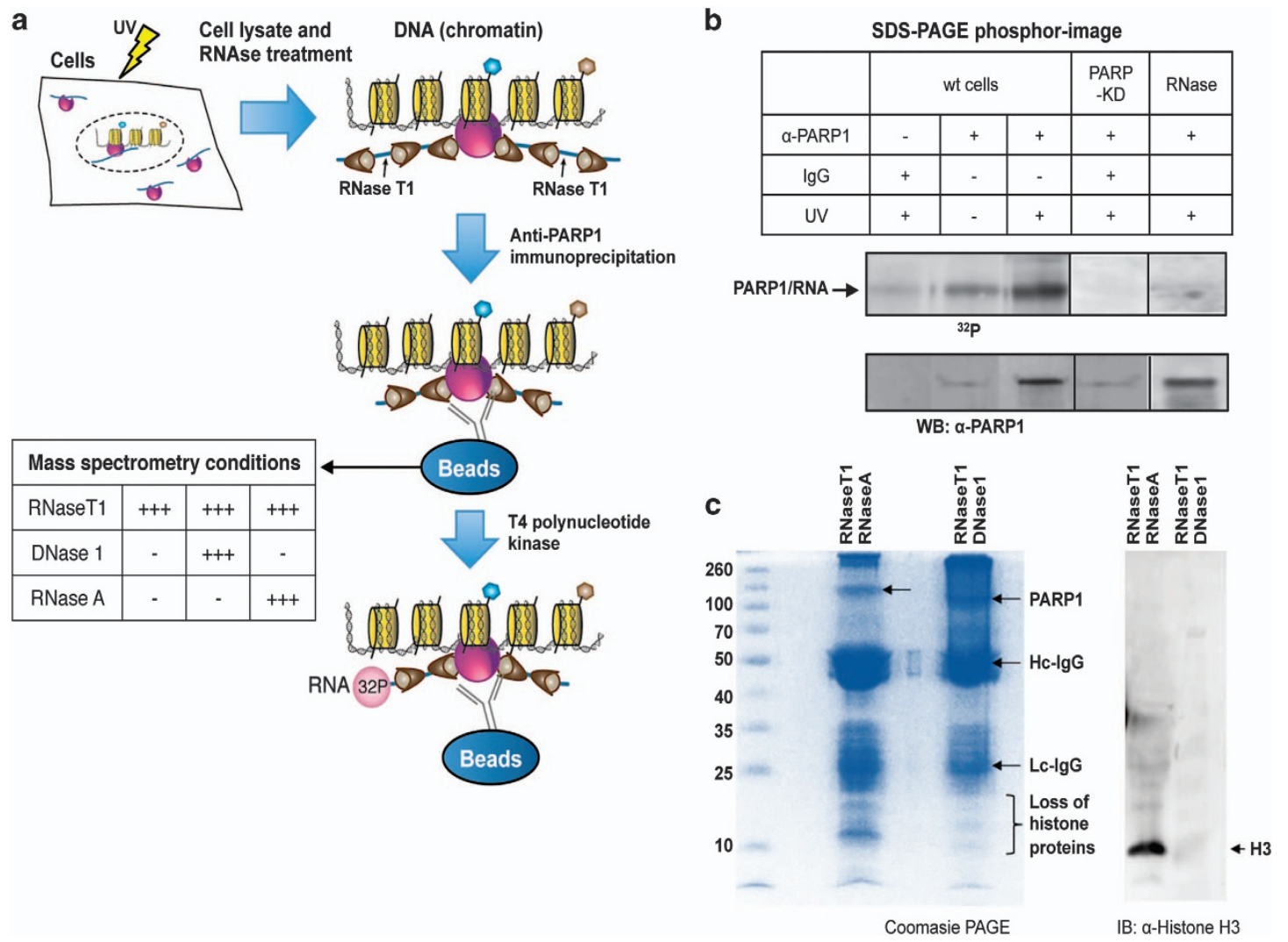

Figure 7 Identification of PARP1 interactome in vivo. PARP1 is covalently cross-linked to nascent RNAs using 365 nm iUV light and 4-thiouridine. PARP1-bound nascent RNAs are immunoprecipitated using PARP1 antibody and then purified under stringent conditions. (a) PAR-CLIP procedure. (b) Radiolabeled PARP1-bound RNAs are blotted onto nitrocellulose, released by proteinase $\mathrm{K}$ and analyzed on a phosphoimager. The same blot was probed with anti-PARP1 antibody confirming PARP1-RNA binding. Knockdown of PARP1 or stringent RNase treatment of immunoprecipitated samples eliminated the PARP1-RNA band. Furthermore, protein samples resulting from PAR-CLIP experiments were split into three aliquots (i) no further treatment; (ii) stringent DNase1 treatment; (iii) stringent RNase A. These samples were subjected to mass spectrometry (Supplementary Table S5). (c) Proteins released from stringent RNase A and DNase1 digests were analyzed on a coomassie-stained gel and also probed with anti-H3 antibody to show depletion of histones.

other PARP1-target regions, no H3K4me3 was found (Supplementary Figure S6C), implying that other factors or histone modifications might also be important for PARP1-nucleosome association. On the other hand, compared with PARP1 KD, no reduction in H3K4me3 occupancy (Figure 6, Lane 3) was observed in PJ34-treated cells as previously reported [46]. We believe the difference could be due to length of PJ34 treatment and/or cell type (S2 cells with one PARP1 and MCF7 cells with several PARP1s). Taken together, these results support our findings of the differential splicing outcomes in PARP1 KD and PARylation-inhibited cells. And, as with the case of PARP1's function in transcription, the physical presence of PARP1 is critical in some molecular pathways, possibly involving the chromatin structure, whereas PARylation is important in others [27, 44, 48].
Third, we hypothesized that PARP1 might act as an adapter bringing RNA to specific regions of chromatin. To test this hypothesis, we first sought to determine whether PARP1 binds RNA in vivo. To this end, PAR-CLIP (photoactivatable cross-linking and immunoprecipitation) protocol [49] (Figure 7a) was used and PARP1-associated RNAs were immunoprecipitated with a PARP1 antibody (Figure $7 \mathrm{a}$ and b). As spliceosome dynamics and composition are very similar between Drosophila and humans [50], and most known human-SFs have orthologues in flies [51], experiments were performed in both $\mathrm{S} 2$ and HeLa cells. Complexes representing the expected molecular weight of a single molecule of PARP1 bound to its target RNAs were observed in both cell types. This band was eliminated in stringent RNase A treatment as well as in PARP1 KD cells (Figure $7 \mathrm{~b}$ and Supplementary 
Figure S7A). Our data from three independent experiments show direct in vivo binding of PARP1 to RNA, supporting previous studies that showed or postulated the binding of PARP1 to RNA and RNA binding proteins [52-54]. In effect, the binding of PARP1 to mRNA at some target exons (Figure 5) was validated after PAR-CLIP in both cell types using RT-PCR (Supplementary Figure S7B). Our inability to validate CAPT/CAP mRNA binding could be due to the differences in conditions used in the different methods (nuc-ChIP-seq versus PAR-CLIP-qRT-PCR).

As PARP1 is a known chromatin-binding protein, we tested the possibility that PARP1 binds both RNA and chromatin at the same time. Following the PARCLIP experiment and after PARP-IP - although the PARP1:RNA complexes were still on beads - samples were subjected to polyacrylamide gel electrophoresis (PAGE) analyses. Gel pieces were excised, trypsindigested and used for tandem mass spectrometry analyses to determine the protein components. We filtered and retained only proteins with a stringent mascot level of $>23$, with more than four unique peptides and present in four independent experiments. We observed the presence of histones, other chromatin proteins and proteins involved in pre-mRNA regulation (Figure 7c and Supplementary Table S5). Based on these results, we believe that PARP1 is in a complex with chromatin, RNA and SFs. Remarkably, the stringent RNase A or DNase I digest of PAR-CLIP samples resulted in the a significant number of RNA or DNA (chromatin) proteins, respectively (Figure 7 and Supplementary Table S5).

Fourth, PARylation has been proposed to assist in the opening of chromatin structure and to increase access of factors to DNA [29, 30, 55]. Given that PARP1 binds chromatin and mRNA (Figure 7) and given that PARylation activates SFs $[37,44,56]$ we speculated that PARP1 might be recruiting SFs. For this purpose, we analyzed the association of PARP1 and the SF 3B subunit 1 (SF3B1), a component of the U2 snRNPs. Owing to the availability of ChIP-grade antibodies, these experiments were carried out in human HeLa cells. NChIP experiments using antibodies specific for SF3B1 were performed and probed for the presence of PARP1. Most SF3B1-bound nucleosomes also contained bound PARP1 as exemplified with SF3B1-ChIP (Figure 8a, Lane 3). Furthermore, in $\mathrm{H} 3$-pulldowns, we observed the presence of some SF3B1, implying that SF3B1 binds only a small subset of all nucleosomes (Figure 8a, Lane 4). PARP1, on the other hand, binds to more nucleosomes, possibly because of its role in other chromatin gene regulatory programs, not necessarily linked to SF3B1. To provide additional evidence for a physical association between PARP1 and SFs, we fractionated HeLa nuclear extracts on a $10-30 \%$ glycerol gradient. We observed PARP1 co-sedimenting with SF3B1 (fractions 6-14 in Supplementary Figure S8), arguing for a stable association between PARP1 and SF3B1. These results are in line with previous studies showing spliceosomal complexes as part of PARP1 interactome [57] and vice versa: PARP1 as one of the interacting proteins in the spliceosomal complex [58]. As PARylation is known to affect protein complex stability [59, 60], we asked whether this PARP1-SF3B1 association requires PARylation. Though PJ34 treatment resulted in a significant decrease in global PARylation levels (Supplementary Figure S3C), this treatment did not abrogate PARP1-SF3B1nucleosome association as measured by ChIP (Figure 8b). Knockdown of PARP1 resulted in less SF3B1 association with nucleosomes, implicating PARP1 in stabilizing the SF3B1-nucleosome complex (Figure 8b). Taken together, our results support the idea that a fraction of PARP1 is in a complex with spliceosomal components, possibly recruiting them to relevant sites important for splicing regulation.

\section{Discussion}

The findings presented here reveal a functional role for PARP1 in the regulation of pre-mRNA splicing. In particular, we have shown that PARP1 binds to nucleosomes at exon/intron boundaries corresponding to specific splice sites. In addition, we demonstrate that knockdown of PARP1 or inhibition of its PARylation activity, leads to changes in specific alternative splicing patterns. Moreover, PARP1 and its PARylation activity have distinct effects on splicing with nucleosomal PARP1 causing direct changes in ASEs, whereas PARylation inhibition resulted in changes in gene expression of SFs, implying an indirect effect on splicing. Our findings thus reveal an important role for PARP1 in regulating alternative splicing, at both the gene-specific and global level. Importantly, we find that in addition to its well-established association with chromatin, PARP1 displays in vivo RNA binding and binds to SF3B1, a member of the U2 spliceosomal complex.

Our studies show also that the effects of PARP1 or PARylation inhibition are quite distinct from each other, with no specific direction in splicing modulation (exon inclusion or exon exclusion). Surprisingly, our analyses showed little overlap between ASEs modulated after PARP1 knockdown and PARylation 


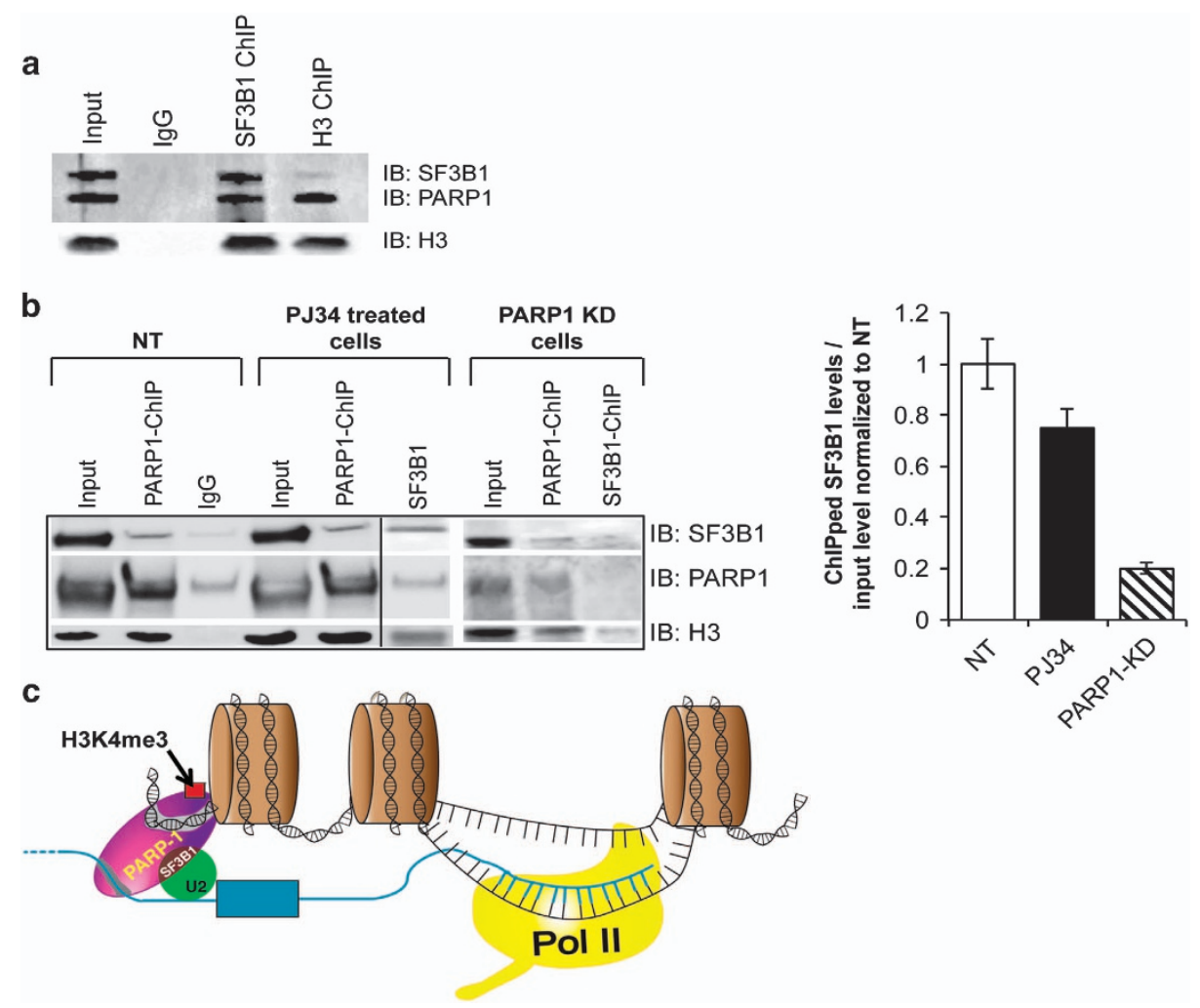

Figure 8 PARP1 regulation of co-transcriptional splicing. (a) PARP1 and SF3B1 bind to the same nucleosomes. SF3B1 (U2 snRNP) ChIP shows that most SF3B1-bound nucleosomes (SF3B-IP) also bind PARP1. However, PARP1 binds other nucleosomes as indicated by H3 ChIP. Knockdown of PARP1 impaired the association of SF3B1 to nucleosomes, whereas inhibition of PARylation had no significant effect. (b) ChIP experiments showing co-occupancy of PARP1 and SF3B1 in HeLa cells. Knockdown of PARP1 resulted in reduction of SF3B1-nucleosome association, whereas PARylation inhibition (PJ34 treatment had no such effect). The results (bar graph) are represented as mean plus s.e.m. $\left(n \geqslant 3\right.$; Student's $t$-test, $\left.{ }^{*} P<0.05\right)$. (c) Model of PARP1 in mediating co-transcriptional splicing. PARP1 binds to specific nucleosomes at exons (specified by specific histone PTMs, for example, H3K4me3) and also binds to the nascent pre-mRNA and recruits SF3B1 a U2 component. U2 binds to the branch-point recognized by the splicing machinery, allowing PARP1 to influence exon recognition. RNA polymerase II generating the nascent pre-mRNA is shown on the right.

inhibition. We hypothesize that these differences are due to several possible reasons:

First, PARP1 knockdown results in a general reduction in PARP1 occupancy over PARP1-target exons, whereas PARylation inhibition does not change PARP1 occupancy (Figure 6). In fact, we observed a slight reproducible increase in PARP1 occupancy after PARylation inhibition, though more studies will be needed to validate this finding. However, these results are in line with previous studies showing that extensive PARylation of PARP1 inhibits its nucleosome binding [61-63]. On the other hand, it is plausible that lack of PARylation results in less-PARP1 degradation, as PARylation negatively regulates certain proteins by dissociating protein complexes or by promoting their ubiquitination and their proteasomal degradation $[59,60]$.
Second, our studies show that with PARP1 knockdown, PARP1 is absent at these exons with a concomitant decrease in $\mathrm{H} 3 \mathrm{~K} 4 \mathrm{me} 3$, whereas with PARylation inhibition, PARP1 and $\mathrm{H} 3 \mathrm{~K} 4 \mathrm{me} 3$ are present. If PARP1 were to function by regulating the deposition of $\mathrm{H} 3 \mathrm{~K} 4 \mathrm{me} 3$ [46], a histone modification implicated in splicing, there would be a difference between the splicing outcomes between PARP1 KD and PARylation inhibition.

Third, PARylation inhibition had no effect on PARP1-SF3B1-nucleosome binding (Supplementary Figure S8A). A similar situation has been shown during transcription, where PARP1 is essential for recruiting the transcription machinery, such as the mediator complex or co-regulators (for example, $\mathrm{p} 300$ ), and its catalytic activity is not required in these processes [64, 65]. 
Our studies showing differential effects on ASEs mediated during PARP1 KD and PARylation inhibition is thought to be a consequence of the absence of PARP in PARP1 knockdown, whereas in PARylation inhibition, PARP1 is still present and can effect changes in ASE. This finding supports distinct roles of PARP1 and PARylation in splicing regulation; a direct effect by PARP1 produced by the presence of the PARP1 protein and an indirect effect produced by PARylation. Indeed PARylation has been shown to have key roles in transcription regulation in some contexts [44, 46, 66], whereas being dispensable in others [67, 68]. A recent study by Muthurajan et al. [48]. shows that PAR on PARP1 switches PARP1 from a chromatin architectural protein to a histone chaperone and nucleosome assembly factor, demonstrating differential functional outcomes between PARP1 as a protein per se and PARP1's catalytic activity. In addition, though the Drosophila genome encodes only one PARP1 protein, it also encodes a tankyrase (PARP5 in humans) with a PARylation activity, therefore the differences in PARP1 knockdown effect versus PARylation inhibition on splicing, could also be masked by functional redundancy.

Finally, the co-presence of PARP1 and H3K4me3, may point to the specificity of PARP1 binding. However, positive correlations genome-wide of PARP1-chromatin-binding with chromatin regions containing other histone modifications such as $\mathrm{H} 3 \mathrm{~K} 36 \mathrm{me} 3$ and H2Av (Figure 2c) that have been implicated in splicing (reviewed in ref. 69) were also observed. It is therefore possible that other histone modifications might also be important in targeting PARP1 to specific regions of the genome. It is therefore possible that PARP1 recognizes specific histone modifications to regulate alternative splicing decisions. Indeed, it has been hypothesized that adapter proteins recognize specific histone modifications and recruit SFs close to chromatin and thus aid in co-transcriptional splicing. The activities of adapter proteins range from activation and repression of transcription, chromatin remodeling, or splicing efficiency as well as other activities [70]. It has been suggested that H3K4me3 serves to facilitate the competency of premRNA maturation through the bridging of spliceosomal components [10]. PARP1, therefore, might act as an adapter associating with $\mathrm{H} 3 \mathrm{~K} 4 \mathrm{me} 3$ at exons and aiding to bridge SFs to functional sites on chromatin.

We propose the following model (Figure 8c): PARP1 binds to nucleosomes containing $\mathrm{H} 3 \mathrm{~K} 4 \mathrm{me} 3$ at exons as well as sites on nascent RNA, and through its association with U2 snRNP regulates usage of alternative exons. In this scenario, PARP1 not only acts as an adapter, ensuring that nascent pre-mRNAs are held close to chromatin [71], but also marks the exons both on chromatin and the nascent pre-mRNAs. Our data (Figure 1) showing PARP1 presence at exons and our ability to amplify some of the PARP1-bound exons from PARP1-bound RNAs (Supplementary Figure S6B) support this idea. Furthermore, PARP1 bound at these exonic sites on pre-mRNA is then able to recruit SFs to these regions. Several observations support this possibility. First, our CLIP-mass spectrometry data show that PARP1 is in a complex with chromatin, and associate with RNA and SFs (Figures 7 and $8 \mathrm{a}$ and $\mathrm{b}$ and Supplementary Figure S7A). Also PARP1 knockdown results in impaired SF3B1nucleosomal recruitment (Figure 8b). Our studies suggest that PARP1 adds another layer of complexity to chromatin modulation in co-transcriptional splicing, and support a model in which PARP1-containing complexes regulate gene expression at both transcription initiation and pre-mRNA-splicing levels. Future studies are important to decipher the mechanism of PARP1 in RNA biogenesis.

\section{Materials and Methods}

\section{S2 and HeLa cell culture}

Drosophila melanogaster S2-DRSC cells (obtained from the Drosophila Genomics Resource Center) were cultured in Schneider's Drosophila medium (Life Technologies, Grand Island, NY, USA) supplemented with $10 \%$ fetal calf serum (Hyclone, Logan, UT, USA). HeLa cells were obtained from the American Type Culture Collection (Rockville, MD, USA) and cultured Dulbecco's modified Eagle's medium containing $1 \mathrm{~mm}$ sodium pyruvate, $0.1 \mathrm{~mm}$ non-essential amino acid, $10 \%$ fetal calf serum, $100 \mathrm{U} / \mathrm{ml}$ penicillin and $100 \mu \mathrm{g} / \mathrm{ml}$ streptomycin at $37^{\circ} \mathrm{C}$ in a humidified environment containing $5 \% \mathrm{CO}_{2}$ and $95 \%$ air.

\section{Drosophila strains}

All Drosophila strains were kept on standard media at $25^{\circ} \mathrm{C}$. wild-type Oregon $\mathrm{R}$ and PARP ${ }^{\mathrm{C} 03256}$ mutant flies [44] were obtained from Professor Tulin. All experiments used cells were experimental samples and controls were growth time and cell-density matched.

\section{ChIP of PARP1-bound nucleosomes}

Chromatin fixation and immunoprecipitation were performed essentially as described by [72]. In brief, $1 \times 10^{7}$ cells were resuspended in PBS and fixed with 1\% formaldehyde for $10 \mathrm{~min}$. Next, cells were washed and pelled cells were resuspended in lysis buffer ( $1 \%$ SDS, $10 \mathrm{~mm}$ EDTA, protease inhibitors, $50 \mathrm{~mm}$ Tris- $\mathrm{HCl},(\mathrm{pH} 8)$ ) for $5 \mathrm{~min}$ on ice. Resulting nuclei were pelleted and washed with MNase buffer (10 mM Tris (pH 7.4), $15 \mathrm{~mm} \mathrm{NaCl}, 60 \mathrm{~mm} \mathrm{KCl}, 0.15 \mathrm{~mm}$ spermine, $0.5 \mathrm{~mm}$ spermidine, $2 \mathrm{~mm} \mathrm{CaCl}_{2}$ ). Chromatin was subjected to micrococcal 
nuclease (MNase) digestion, with varying concentrations of MNase concentration, in MNase buffer at RT to yield nucleosomal fragments. The addition of $25 \mathrm{~mm}$ EDTA and $0.2 \%$ SDS stopped the reactions. Cellular debris was pelleted and the supernatant recovered. Lysates were diluted 1:10 in ChIP dilution buffer $(0.01 \%$ SDS, $1.1 \%$ Triton X-100, 1.2 mм EDTA, $167 \mathrm{~mm} \mathrm{NaCl}$, protease inhibitors, $16.7 \mathrm{~mm}$ Tris- $\mathrm{HCl}$, (pH 8). Non-specific background was removed by incubating the MNase digested chromatin with Protein A/G dynabeads (Invitrogen, Carlsbad, CA, USA) overnight at $4{ }^{\circ} \mathrm{C}$ with rotation in the presence of BSA $\left(250 \mu \mathrm{g} \mathrm{ml}^{-1}\right)$. Precleared chromatin solutions were incubated overnight at $4{ }^{\circ} \mathrm{C}$ with rotation with antibodies against PARP1 (\#39559, Active Motif, Carlsbad, CA, USA), H3 (ab1791, Abcam, Cambridge, MA, USA) and for control, immunoglobulin G (I8140; Sigma-Aldrich, St Louis, MO, USA). For quality control, $100 \mu 1$ of the precleared chromatin was purified by QIAquick PCR Purification Kit (Qiagen, Germantown, MD, USA) and DNA fragment sizes were analyzed and confirmed to correspond to one to three nucleosome fragments. For the ChIP samples, the complex was washed and eluted, and immunoprecipitated material was purified using the QIAquick PCR Purification Kit (Qiagen). The purified DNA was analyzed by $\mathrm{qPCR}$ with respect to input using GelStar (Lonza, Walkersville, MD, USA)) and Taq DNA Polymerase (Invitrogen).

Quantitative real-time PCR was used to measure PARP1, nucleosome and H3K4me3 occupancy. ChIP-qPCR experiments were done with the following antibodies: PARP1, H3 and $\mathrm{H} 3 \mathrm{~K} 4 \mathrm{me} 3$ to measure for PARP1, nucleosome and H3K4me3 occupancy according to ref 72. PCR signals from immunoprecipitated samples were normalized to input and in some cases to the total histone $\mathrm{H} 3$ control.

\section{Nuc-ChIP-seq and MNase-seq data analyses}

Nuc-ChIP-seq was performed following the protocol described in our recent publication [28]. As no peak-calling program has gained consensus acceptance by the scientific community as the preferred tool for ChIP-Seq data analysis [73], we used two different methods to analyze the sequenced reads.

Method 1 Nucleosomes were defined as described in [74, 75]. To define nuc-ChIP- seq and MNase-seq centers based on the single-end reads we first estimated the average DNA insert length, $d$. We calculated the cross-correlation of the tag start positions between the reads on the Watson and Crick strands and identified the peaks at $d=156$ and $d=150$ for the PARP1bound nucleosomes and total nucleosomes, respectively. Thus, the tags on the Watson and Crick strands were shifted by $d / 2$ toward $3^{\prime}$ and $5^{\prime}$, respectively, so that the tag frequency on position $i$ can reflect the probability of position $i$ being the PARP1-bound nucleosome or total nucleosome centers. The relative abundance of PARP1-bound nucleosomes was estimated by counting the number of PARP1-ChIP-seq nucleosome centers within each region and dividing that by the total number of mapped reads. Custom scripts were used to calculate the nucleosome center as previously done [28, 74, 75].

Method 2 Model-based Analysis for ChIP-Seq (MACS) was used to identify peaks from the nuc-ChIP-Seq data as follows.
For each replicate, a default $P$-value significance of $1 \times 10^{-5}$ was defined as significant. ChIP-Seq peaks were detected using Macs2 [76, 77], with the broad option and a window size of 200 bp. Overlaps between each ChIP-Seq data peaks and PARP1 peaks were determined by using an overlap of at least $10 \%$ of the ChIP-Seq peak with the PARP1 peak. Both methods yielded very similar results in spite of having different limitations: method 1 produces background noise and method 2 possibly eliminates PARP1 binding sites. To visualize PARP1-bound nucleosome tag-density across the human chromosomes on the UCSC browser for hg19, ready-tovisualize bedgraph files were created using the HOMER package v3.13 [78]. In brief, aligned reads were extended to the average fragment size (109-160 bp) and read coverage on each base across the genome was calculated. Read coverage was then scaled to one million and normalized with the total number of reads. All publicly available data used for pairwise comparisons with our PARP1 data were processed in the same way. We also used total nucleosome (nucleosome-seq) data to normalize for background correction.'

\section{Native ChIP (NChIP) of SF3B1-bound nucleosomes}

NChIP was performed similarly to nuc-ChIP described above albeit with no fixation step. In this protocol, antibodies used were SF3B1, PARP1 and H3 antibodies.

\section{HeLa cell extract fractionation}

HeLa cell extract fractionation was done according to ref. 79. In brief, nuclear extract from HeLa cells was loaded onto a linear 4-ml 15-30\% glycerol gradient prepared in G150 buffer (20 mм HEPES, pH 7.9, 150 mм NaCl, $1.5 \mathrm{~mm} \mathrm{MgCl}_{2}, 0.5 \mathrm{~mm}$ dithioerythritol). Gradient was centrifuged in a Beckman SW60Ti rotor at $35000 \mathrm{rpm}$, at $4{ }^{\circ} \mathrm{C}$ for $15 \mathrm{~h}$. Thereafter, the gradient was fractionated into 26 fractions of $150 \mu \mathrm{l}$ each. In total, $30 \mu \mathrm{l}$ aliquots from the fractionated samples were loaded onto $10 \%$ SDS-PAGE. To estimate the Svedberg (S) values, we used conalbumin (molecular weight $75000 \mathrm{D} ; 5.4 \mathrm{~S}$ ), aldolase (15 $800 \mathrm{D}, 11.5 \mathrm{~S})$ and ferritin $(440000,17 \mathrm{~S})$, all from GE healthcare, Piscataway, NJ, USA.

\section{siRNA production and treatment of cells}

PCR product made against specific exons of PARP1 (siRNA1 for KD1) was obtained from the Lis laboratory Cornell University, Ithaca, NY, USA [29]. PCR products made against the specific exons of PARP1 (siRNA2) and LacZ were obtained from the Drosophila RNAi Screening Center (FlyRNAi.org - the database of the Drosophila RNAi screening center: 2012 update) to produce double-stranded RNA (dsRNA) for PARP1 knockdown and non-targeting control LacZ. PCR products were further amplified and dsRNA was produced and purified according to the manufacturer MEGAscript RNAi Kit (Life Technologies). RNAi treatments were as follows: S2 cells were treated with dsRNA, produced according to the Ambion MEGAscript manual. To achieve efficient RNAi knockdown ( $>30 \%$ ), concentrations of $\sim 10 \mathrm{~nm}$ of dsRNA (siRNA) were used. siRNA targeting the coding sequence of $\beta$-galactosidase (LacZ) was used as a non-specific control. SiRNAs against human PARP1 (ON-TARGETplus) 
from Thermo Scientific Dharmacon and transfection was performed as per the manufacturer using Dharmafect reagent 2.

\section{Inhibition of PARPI in D. melanogaster cells}

PJ34 was added to $\mathrm{S} 2$ cells in media, at final concentrations of $300 \mathrm{~nm}$ and $10 \mu \mathrm{M}$ and incubated further for $10 \mathrm{~h}$ at growth temperature [29, 38]. Cells were harvested for total RNAs extraction. Inhibition of PARylation was validated using Trevigen's Pharmacodynamic Assay II (4520-096-K) according to manufacturer's instructions.

\section{Antibodies}

The following antibodies were used in this study: CHIP-grade PARP1 (Active Motif: 39559), CHIP-grade H3 antibody (Abcam: ab1791), CHIP-grade H3K4me3 antibody (Abcam: ab1791) and CHIP-grade custom-made SF3B1, which was a kind gift from the Stamm laboratory, University of Kentucky, Lexington, KY, USA.

\section{$P A R-C L I P$}

PAR-CLIP was performed according to ref. 49. Samples were run on $8-20 \%$ PAGE gel (Invitrogen) and transferred on nitrocellulose membrane before visualization on a phosphoimager. Segments representing PARP1-bound RNAs were excised from membrane, purified and reverse transcribed into complementary DNA (cDNA) using qScript cDNA Supermix (Quanta Biosciences, Gaithersburg, MD USA).

\section{Analyses of gene expression data}

Drosophila genome TSSs, transcription stop ends, exon/ intron junction data and gene expression data were downloaded from http://genome.ucsc.edu/cgi-bin/hgTables and http://inter mine.modencode.org, respectively, and processed as below:

\section{$R N A$-seq data analyses}

A total of six fastq files were analyzed in this study, each representing a separate sample (two control; two PARP1 KD, and two PARylation). These fastq files were examined for quality control using FastQC [80]. Examination of the results of FastQC indicated that no further pre-processing was necessary. The RNA-seq reads were then processed using the Tuxedo suite [39]. The first step mapped reads to the Drosophila reference genome $(\mathrm{dm} 3)$ using TopHat2 v2.0.10 [81] with the multiprocessor option - p 5 and the remaining parameters as the TopHat2 defaults, allowing at most two mismatches to the reference. In this analysis, we compared Control with PARP1 KD and PARylation. Transcripts were assembled using Cufflinks v2.1.1 [39]. Aligned reads were used as inputs into Cuffdiff2 [39] to detect DEGs, transcripts and ASEs along with their abundances, guided by the flyBase dm3 gtf track downloaded from the UCSC Genome Browser (http://genome. ucsc.edu). A min-alignment-count of seven reads was used as the threshold of the minimum number of reads that map to each transcript, and a $P$-value $\leqslant 0.05$ was used to determine differentially expressed (DE) events. FPKM (Fragments Per Kilobase per Million mapped reads) correlation analysis showed high correlation between the two control samples (Pearson correlation of $r=0.85 ; \quad P=3.14 \times 10^{-13}$ ), between the two
PARP1-knockdown samples (Pearson correlation of $r=0.985$; $\left.P=5.29 \times 10^{-21}\right)$ and between the two PARylation inhibition samples (Pearson correlation of $r=0.980 ; P=9.37 \times 10^{-33}$ ), indicating a very good transcript expression correlation between replica libraries.

ASEs using MATS MATS [41] was used to measure ASEs. Results were generated for five events: SE, mutually exclusive exons, alternative $3^{\prime}$ splice sites, alternative $5^{\prime}$ splice sites and retained introns. The cutoff splicing default difference is 0.0001 for $0.01 \%$ difference. Thus, the valid cutoff used is $0 \leqslant$ cutoff $<1$. Second, AS events whose gene expression levels differed more than the given cutoff fold change between the two samples were filtered out. Valid: fold change $>1.0$. The default is 10000.0

Alternative splicing analysis Using MISO Alternative event annotations for $D$. melanogaster were downloaded from the MISO annotations page [42]. First, alternative event annotations were indexed using the index_gff module, which is part of the MISO toolkit. We ran MISO to get isoform expression estimates. In this step, Psi values were computed for each sample. We then made pairwise comparisons between samples to detect DE isoforms/events. In this step, Bayes factors and delta Psi values were computed between the samples. The last step was to filter DE events based on different criteria. Given a MISO Bayes factor comparison file for two-isoform events, events can be filtered based on their coverage or magnitude of change. We filtered our events based on the following parameters: (a) at least 1 inclusion read (-num-inc 1); (b) at least 1 exclusion read (-num-exc 1); (c) the sum of inclusion and exclusion reads is at least 10 (-num-sum-incexc10); (d) the $\Delta \Psi$ is at least 0.20 (-delta-psi 0.20); (e) the Bayes factor is at least 10 (-bayes-factor 10).

Comparing RNA-seq PARP1/PARylation-mediated ASES and PARPI binding from nuc-ChIP-seq

We used Bowtie version 1.0.1 to map the read sequences to the Drosophila genome $\mathrm{dm} 3$. We then used MACS (version 1.4.2 to call peaks for each replicate (and combined) PARP1binding site. We counted the number of peaks corresponding to each event, by determining the overlap between each peak file (PARP1 binding) with each event file (ASE). To determine whether there were PARP1-binding sites associated with the significant ASEs generated by MATS, we computed the number of peaks (per replicate and combined) that correspond to each event for each comparison.

\section{Liquid chromatography-electrospray ionization-tandem mass spectrometry analysis}

After PAR-CLIP, samples on the beads were subjected to SDS loading buffer and PAGE electrophoresis as follows: onethird of IP samples were used directly for mass spectrometry to determine the protein complex associated with PARP1 RNA. The other third was subjected to stringent RNase A digest and the last third was subjected to stringent DNase1 digest. These latter samples were also used for (MS/MS) mass spectrometry analyses, followed by scanning with Mascot as previously reported [82]. Peptide matches that pass the filter associated with 
the strict False Discovery Rate (with target setting of 0.01) are assigned as high confidence. For MS/MS ion search, proteins with two or more high confidence peptides were considered unambiguous identifications without manual inspection. Proteins identified with one high confidence peptide were manually inspected and confirmed. To generate the list of proteins, we filtered the peptides retrieved from the database, leaving only those with individual Mascot scores above 23 and eliminating any consistent matches with $\mathrm{IgG}$ control sample as a background.

LC-MS/MS analysis was performed using an LTQ-Orbitrap mass spectrometer (Thermo Fisher Scientific, Waltham, MA, USA) coupled with an Eksigent Nanoflex cHiPLC system (Eksigent, Dublin, CA, USA) through a nano-electrospray ionization source. The peptide samples were separated with a reversed phase cHiPLC column $(75 \times 150 \mathrm{~mm})$ at a flow rate of $300-\mathrm{nl} / \mathrm{min}$. The mobile phase A was water with $0.1 \%(v / v)$ formic acid, whereas B was acetonitrile with $0.1 \%(v / v)$ formic acid. A 50-min gradient condition was applied: initial 3\% mobile phase B was increased linearly to $50 \%$ in $24 \mathrm{~min}$ and further to $85 \%$ and $95 \%$ for 5 min each before it was decreased to $3 \%$ and re-equilibrated. The mass analysis method consisted of one segment with eight scan events. The first scan event was an Orbitrap MS scan $(100-1600 \mathrm{~m} / \mathrm{z})$ with 60000 resolution for parent ions followed by data dependent MS/MS for fragmentation of the seven most intense ions with collision-induced dissociation method.

\section{MS/MS protein identification}

The LC-MS/MS data were submitted to a local mascot server for MS/MS protein identification via Proteome Discoverer (version 1.3, Thermo Fisher Scientific) against Homo sapiens (human) protein sequences contained within the Swissprot database. Typical parameters used in the Mascot MS/MS ion search were: trypsin digest with maximum of two miscleavages, cysteine carbamidomethylation, methionine oxidation, a maximum of 10 p.p.m. MS error tolerance and a maximum of 0.8 Da MS/MS error tolerance. A decoy database was built and searched. Filter settings that determine false discovery rates (FDR) are used to distribute the confidence indicators for the peptide matches. Peptide matches that pass the filter associated with the strict FDR (with target setting of 0.01) are assigned as high confidence. For MS/MS ion search, proteins with two or more high confidence peptides were considered unambiguous identifications without manual inspection. Proteins identified with one high confidence peptide were manually inspected and confirmed.

\section{Validation of isoform expression}

Real-time quantitative reverse transcriptase-PCR analysis Real-time PCR was performed in a total volume of $20 \mu \mathrm{l}$ including $2 \mu \mathrm{l}$ of cDNA, primers $(0.2 \mathrm{~mm}$ each) and $10 \mu \mathrm{l}$ of SYBR Green mix (Roche, Mannheim, Germany) using primer sets (Supplementary Table S6). Reactions were run on a Biorad CFX 96 machine using the following cycling parameters $\left(95^{\circ} \mathrm{C}\right.$ for $5 \mathrm{~min}, 35$ cycles of $10 \mathrm{~s}$ at $95^{\circ} \mathrm{C}, 20 \mathrm{~s}$ at $60^{\circ} \mathrm{C}$ and $15 \mathrm{~s}$ at $72^{\circ}$ $\mathrm{C}$; melting curve: $10 \mathrm{~s}$ at $95^{\circ} \mathrm{C}, 60 \mathrm{~s}$ at $60^{\circ} \mathrm{C}$ ). For qualitycontrol purposes, melting curves for all samples were acquired.
Transcripts levels were normalized to Actb and fold change was quantified using the standard curve method. The relative expression of each gene calculated as a fold difference between treated and untreated samples. Experiments were conducted in triplicate with at least three biological replicates.

Semi-quantitative PCR to measure isoform expression mRNA expression analyses were performed as described [83]. In brief, total RNAs were isolated (QIAGEN RNeasy) (Qiagen, Vinlo, the Netherlands) from different experimental cells and digested with DNase1 prior to reverse-transcription reaction with qScript cDNA Supermix (Quanta Biosciences). Also total RNA from 0-12 h embryos from flies were isolated. The resultant cDNAs were used in semi-quantitative PCR with the indicated primer sets (Supplementary Table S6) and gels were stained with GelStar (Lonza). PCR cycling parameters were $95^{\circ} \mathrm{C}$ for $5 \mathrm{~min}$, 23 cycles of $10 \mathrm{~s}$ at $95^{\circ} \mathrm{C}, 20 \mathrm{~s}$ at $55^{\circ} \mathrm{C}$ (depending on the annealing temperature of the primers), and $15 \mathrm{~s}$ at $72{ }^{\circ} \mathrm{C} ; 72^{\circ} \mathrm{C}$ for $5 \mathrm{~min}$ and $4{ }^{\circ} \mathrm{C}$ forever. PCR products were resolved on $2 \%$ agarose gels and stained with GelStar Nucleic Acid Gel Stain. Splice isoforms were confirmed by cloning the products from PCR analyses using Topo blunt end cloning Kit (Invitrogen) according to manufacturer's protocol and sequenced using the sanger method. At last, some of the detected transcripts are not annotated and were confirmed by sanger sequencing. Products from neighboring constitutive exons of the same genes were used to standardize for total transcription.

\section{In silico analysis}

The following splice site prediction programs were used to predict the effect of variants on the efficiency of splicing: GeneSplicer (http://www.cbcb.umd.edu/software/GeneSplicer); Splice Site Prediction by Neural Network (http://www.fruitfly. org/seq_tools/splice.html); NNSPLICE 0.9 version (http://www. fruitfly.org/seq_tools/splice.html); and Drosophila Melanogaster Exon Database (http://proline.bic.nus.edu.sg/dedb/cgi-bin/viewer. py?id=13068). In silico PCR program, http://genome.ucsc. edu/index.html?org $=$ Human \&db $=$ hg18\&hgsid $=142437216$ was used to first obtain the predicted amplicon sizes before experimental PCR reactions were performed.

\section{Western blots}

Western blots were performed per standard protocol and input dilutions were used as a quantitative indication of signal linearity. In brief, protein samples were resuspended in a reduced sample buffer 9 conatining SDS), and then electrophoresed on a Tris-glycine gel with Tris running buffer; blotted to PVDF membrane; and sequentially probed with primary antibodies against various proteins. Western blot-based detection was performed using alkaline phosphatase-coupled secondary antibodies with Vistra ECF for visualization, and images were obtained using a Typhoon 9400. ImageQuant 5.2 software was used to quantity of proteins in arbitrary units and relative protein concentrations were standardized to $\beta$-actin levels.

\section{Accession numbers}

Data analyzed have been deposited in GEO with accession numbers GSE56120 and GSE56073. 


\section{Conflict of Interest}

The authors declare no conflict of interest.

\section{Acknowledgements}

We thank the Northwestern University Genomic Core and the University of Southwestern Texas Genomic Facility for DNA and RNA sequencing, and the University of Kentucky Small Molecule Mass Spectrometry Core Laboratory for mass spectrometric analyses. We also thank Dr. Louis Hersh. This research was supported by NIH grants P20 GM103436 (ECR); 2P20 RR020171, 1RO1ES024478, NSF and IRSF grant (YNF-M).

\section{Author contributions}

Conceived and designed the experiments: YNF-M. Performed the experiments: EM, JM, PC. Analyzed the data: QZ, JC, J-PW, ANE, ECR, YNF-M. Contributed reagents/ materials: VI, SS, YNF-M. Wrote the paper: EM, JM, PC, VI, SS, ECR, JW, YNF-M.

\section{References}

1 Lopez AJ. Alternative splicing of pre-mRNA: developmental consequences and mechanisms of regulation. Annu Rev Genet 1998; 32: 279-305.

2 Pan Q, Shai O, Lee LJ, Frey BJ, Blencowe BJ. Deep surveying of alternative splicing complexity in the human transcriptome by high-throughput sequencing. Nat Genet 2008; 40: 1413-1415.

3 Wang ET, Sandberg R, Luo S et al. Alternative isoform regulation in human tissue transcriptomes. Nature 2008; 456: 470-476.

4 Venables JP, Tazi J, Juge F. Regulated functional alternative splicing in Drosophila. Nucleic Acids Res 2012; 40: $1-10$.

5 Kelemen O, Convertini P, Zhang $\mathrm{Z}$ et al. Function of alternative splicing. Gene 2013; 514: 1-30.

6 Wang GS, Cooper TA. Splicing in disease: disruption of the splicing code and the decoding machinery. Nat Rev Genet 2007; 8: 749-761.

7 Schwartz S, Ast G. Chromatin density and splicing destiny: on the cross-talk between chromatin structure and splicing. EMBO J 2010; 29: 1629-1636.

8 Allemand E, Batsche E, Muchardt C. Splicing, transcription, and chromatin: a menage a trois. Curr Opin Genet Dev 2008; 18: 145-151.

9 Loomis RJ, Naoe Y, Parker JB et al. Chromatin binding of SRp20 and ASF/SF2 and dissociation from mitotic chromosomes is modulated by histone $\mathrm{H} 3$ serine 10 phosphorylation. Mol Cell 2009; 33: 450-461.

10 Sims RJ 3rd, Millhouse S, Chen CF et al. Recognition of trimethylated histone $\mathrm{H} 3$ lysine 4 facilitates the recruitment of transcription postinitiation factors and pre-mRNA splicing. Mol Cell 2007; 28: 665-676.
11 Barski A, Cuddapah S, Cui K et al. High-resolution profiling of histone methylations in the human genome. Cell 2007; 129: 823-837.

12 Kornblihtt AR, Schor IE, Allo M, Blencowe BJ. When chromatin meets splicing. Nat Struct Mol Biol 2009; 16: 902-903.

13 Schones DE, Zhao K. Genome-wide approaches to studying chromatin modifications. Nat Rev Genet 2008; 9: 179-191.

14 Valouev A, Johnson SM, Boyd SD, Smith CL, Fire AZ, Sidow A. Determinants of nucleosome organization in primary human cells. Nature 2011; 474: 516-520.

15 Kaplan N, Moore IK, Fondufe-Mittendorf Y et al. The DNA-encoded nucleosome organization of a eukaryotic genome. Nature 2009; 458: 362-366.

16 Segal E, Widom J. What controls nucleosome positions? Trends Genet 2009; 25: 335-343.

17 Gelfman S, Cohen N, Yearim A, Ast G. DNA-methylation effect on cotranscriptional splicing is dependent on GC architecture of the exon-intron structure. Genome Res 2013; 23: 789-799.

18 Jansen K, De Groote F, Aerts W, De Schutter J, Duysens J, Jonkers I. Altering length and velocity feedback during a neuro-musculoskeletal simulation of normal gait contributes to hemiparetic gait characteristics. J Neuroeng Rehabil 2014; 11: 78.

19 Luco RF, Pan Q, Tominaga K, Blencowe BJ, Pereira-Smith OM, Misteli T. Regulation of alternative splicing by histone modifications. Science 2010; 327: 996-1000.

20 Amit M, Donyo M, Hollander D et al. Differential GC content between exons and introns establishes distinct strategies of splice-site recognition. Cell Rep 2012; 1: 543-556.

21 Fox-Walsh KL, Dou Y, Lam BJ, Hung SP, Baldi PF, Hertel KJ. The architecture of pre-mRNAs affects mechanisms of splice-site pairing. Proc Natl Acad Sci USA 2005; 102: 16176-16181.

22 Kolasinska-Zwierz P, Down T, Latorre I, Liu T, Liu XS, Ahringer J. Differential chromatin marking of introns and expressed exons by H3K36me3. Nat Genet 2009; 41: 376-381.

23 Spies N, Nielsen CB, Padgett RA, Burge CB. Biased chromatin signatures around polyadenylation sites and exons. Mol Cell 2009; 36: 245-254.

24 Andersson R, Enroth S, Rada-Iglesias A, Wadelius C, Komorowski J. Nucleosomes are well positioned in exons and carry characteristic histone modifications. Genome Res 2009; 19: 1732-1741.

25 Ame JC, Spenlehauer C, de Murcia G. The PARP superfamily. BioEssays 2004; 26: 882-893.

26 Tulin A, Stewart D, Spradling AC. The Drosophila heterochromatic gene encoding poly(ADP-ribose) polymerase (PARP) is required to modulate chromatin structure during development. Genes Dev 2002; 16: 2108-2119.

27 Krishnakumar R, Gamble MJ, Frizzell KM, Berrocal JG, Kininis M, Kraus WL. Reciprocal binding of PARP-1 and 
histone $\mathrm{H} 1$ at promoters specifies transcriptional outcomes. Science 2008; 319: 819-821.

28 Duerinck S, Hagman F, Jonkers I, Van Roy P, Vaes P. Forefoot deformation during stance: does the forefoot collapse during loading? Gait Posture 2014; 39: 40-47.

29 Petesch SJ, Lis JT. Rapid, transcription-independent loss of nucleosomes over a large chromatin domain at Hsp70 loci. Cell 2008; 134: 74-84.

30 Petesch SJ, Lis JT. Overcoming the nucleosome barrier during transcript elongation. Trends Genet 2012; 28: 285-294.

31 Huang, da W, Sherman BT, Lempicki RA. Systematic and integrative analysis of large gene lists using DAVID bioinformatics resources. Nat Protoc 2009; 4: 44-57.

32 Zhang J, Kuo CC, Chen L. GC content around splice sites affects splicing through pre-mRNA secondary structures. BMC Genomics 2011; 12: 90.

33 Tillo D, Hughes TR. G+C content dominates intrinsic nucleosome occupancy. BMC Bioinformatics 2009; 10: 442.

34 Schwartz S, Meshorer E, Ast G. Chromatin organization marks exon-intron structure. Nat Struct Mol Biol 2009; 16: 990-995.

35 Kharchenko PV, Alekseyenko AA, Schwartz YB et al. Comprehensive analysis of the chromatin landscape in Drosophila melanogaster. Nature 2011; 471: 480-485.

36 Gagne JP, Hunter JM, Labrecque B, Chabot B, Poirier GG. A proteomic approach to the identification of heterogeneous nuclear ribonucleoproteins as a new family of poly(ADP-ribose)-binding proteins. Biochem $J$ 2003; 371: 331-340.

37 Ji Y, Tulin AV. Post-transcriptional regulation by poly (ADP-ribosyl)ation of the RNA-binding proteins. Int $\mathrm{J} \mathrm{Mol}$ Sci 2013; 14: 16168-16183.

38 Tapodi A, Debreceni B, Hanto K et al. Pivotal role of Akt activation in mitochondrial protection and cell survival by poly(ADP-ribose)polymerase-1 inhibition in oxidative stress. J Biol Chem 2005; 280: 35767-35775.

39 Trapnell C, Roberts A, Goff L et al. Differential gene and transcript expression analysis of RNA-seq experiments with TopHat and Cufflinks. Nat Protoc 2012; 7: 562-578.

40 Flight RM, Harrison BJ, Mohammad F et al. category Compare, an analytical tool based on feature annotations. Front Genet 2014; 5: 98.

41 Shen S, Park JW, Huang J et al. MATS: a Bayesian framework for flexible detection of differential alternative splicing from RNA-Seq data. Nucleic Acids Res 2012; 40: e61.

42 Katz Y, Wang ET, Airoldi EM, Burge CB. Analysis and design of RNA sequencing experiments for identifying isoform regulation. Nat Methods 2010; 7: 1009-1015.

43 Alamancos GP, Agirre E, Eyras E. Methods to study splicing from high-throughput RNA sequencing data. Methods Mol Biol 2014; 1126: 357-397.

44 Kotova E, Jarnik M, Tulin AV. Uncoupling of the transactivation and transrepression functions of PARP1 protein. Proc Natl Acad Sci USA 2010; 107: 6406-6411.
45 Weber CM, Ramachandran S, Henikoff S. Nucleosomes are context-specific, H2A.Z-modulated barriers to RNA polymerase. Mol Cell 2014; 53: 819-830.

46 Krishnakumar R, Kraus WL. PARP-1 regulates chromatin structure and transcription through a KDM5B-dependent pathway. Mol Cell 2010; 39: 736-749.

47 Nalabothula N, Al-Jumaily T, Eteleeb AM et al. Genomewide profiling of PARP1 reveals an interplay with gene regulatory regions and DNA methylation. PloS ONE 2015; 10: $\mathrm{e} 0135410$.

48 Muthurajan UM, Hepler MR, Hieb AR et al. Automodification switches PARP-1 function from chromatin architectural protein to histone chaperone. Proc Natl Acad Sci USA 2014; 111: 12752-12757.

49 Hafner M, Landthaler M, Burger L et al. PAR-CliP--a method to identify transcriptome-wide the binding sites of RNA binding proteins. J Vis Exp 2010; 41: 2034.

50 Herold N, Will CL, Wolf E, Kastner B, Urlaub H, Luhrmann R. Conservation of the protein composition and electron microscopy structure of Drosophila melanogaster and human spliceosomal complexes. Mol Cell Biol 2009; 29: 281-301.

51 Park JW, Parisky K, Celotto AM, Reenan RA, Graveley BR. Identification of alternative splicing regulators by RNA interference in Drosophila. Proc Natl Acad Sci USA 2004; 101: 15974-15979.

52 Guetg C, Scheifele F, Rosenthal F, Hottiger MO, Santoro R. Inheritance of silent rDNA chromatin is mediated by PARP1 via noncoding RNA. Mol Cell 2012; 45: 790-800.

53 Jungmichel S, Rosenthal F, Altmeyer M, Lukas J, Hottiger MO, Nielsen ML. Proteome-wide identification of poly(ADP-Ribosyl)ation targets in different genotoxic stress responses. Mol Cell 2013; 52: 272-285.

54 Leung AK, Vyas S, Rood JE, Bhutkar A, Sharp PA, Chang P. Poly(ADP-ribose) regulates stress responses and microRNA activity in the cytoplasm. Mol Cell 2011; 42: 489-499.

55 Petesch SJ, Lis JT. Activator-induced spread of poly (ADP-ribose) polymerase promotes nucleosome loss at Hsp70. Mol Cell 2012; 45: 64-74.

56 Ji Y, Tulin AV. Poly(ADP-ribosyl)ation of heterogeneous nuclear ribonucleoproteins modulates splicing. Nucleic Acids Res 2009; 37: 3501-3513.

57 Isabelle M, Moreel X, Gagne JP et al. Investigation of PARP-1, PARP-2, and PARG interactomes by affinity-purification mass spectrometry. Proteome Sci 2010; 8: 22.

58 Ishaq M, Jan FA, Khan MA et al. Effect of mercury and arsenic from industrial effluents on the drinking water and comparison of the water quality of polluted and non-polluted areas: a case study of Peshawar and Lower Dir. Environ Monit Assess 2013; 185: 1483-1494.

59 Ju BG, Solum D, Song EJ et al. Activating the PARP-1 sensor component of the groucho/ TLE1 corepressor complex mediates a CaMKinase IIdelta-dependent 
neurogenic gene activation pathway. Cell 2004; 119: 815-829.

60 Wang Z, Michaud GA, Cheng Z et al. Recognition of the iso-ADP-ribose moiety in poly(ADP-ribose) by WWE domains suggests a general mechanism for poly (ADP-ribosyl)ation-dependent ubiquitination. Genes Dev 2012; 26: 235-240.

61 Kim MY, Mauro S, Gevry N, Lis JT, Kraus WL. NAD +-dependent modulation of chromatin structure and transcription by nucleosome binding properties of PARP-1. Cell 2004; 119: 803-814.

62 Wacker DA, Frizzell KM, Zhang T, Kraus WL. Regulation of chromatin structure and chromatindependent transcription by poly(ADP-ribose) polymerase1: possible targets for drug-based therapies. Subcell Biochem 2007; 41: 45-69.

63 Wacker DA, Ruhl DD, Balagamwala EH, Hope KM, Zhang T, Kraus WL. The DNA binding and catalytic domains of poly(ADP-ribose) polymerase 1 cooperate in the regulation of chromatin structure and transcription. Mol Cell Biol 2007; 27: 7475-7485.

64 Bozzetti F, Santarpia L, Pironi L et al. The prognosis of incurable cachectic cancer patients on home parenteral nutrition: a multi-centre observational study with prospective follow-up of 414 patients. Ann Oncol 2014; 25 : 487-493.

65 Legrand AJ, Choul-Li S, Spriet C et al. The level of Ets-1 protein is regulated by poly(ADP-ribose) polymerase-1 (PARP-1) in cancer cells to prevent DNA damage. PloS ONE 2013; 8: e55883.

66 Cohen-Armon M, Visochek L, Rozensal D et al. DNA-independent PARP-1 activation by phosphorylated ERK2 increases Elk1 activity: a link to histone acetylation. Mol Cell 2007; 25: 297-308.

67 D'Amours D, Desnoyers S, D'Silva I, Poirier GG. Poly (ADP-ribosyl)ation reactions in the regulation of nuclear functions. Biochem J 1999; 342 (Pt 2): 249-268.

68 Hassa PO, Hottiger MO. The diverse biological roles of mammalian PARPS, a small but powerful family of poly-ADP-ribose polymerases. Front Biosci 2008; 13: 3046-3082.

69 Luco RF, Allo M, Schor IE, Kornblihtt AR, Misteli T. Epigenetics in alternative pre-mRNA splicing. Cell 2011; 144: 16-26.

70 Vermeulen M, Timmers HT. Grasping trimethylation of histone H3 at lysine 4. Epigenomics 2010; 2: 395-406.

71 Carrillo Oesterreich F, Preibisch S, Neugebauer KM. Global analysis of nascent RNA reveals transcriptional pausing in terminal exons. Mol Cell 2010; 40: 571-581.

72 Nalabothula N, McVicker G, Maiorano J, Martin R, Pritchard JK, Fondufe-Mittendorf YN. The chromatin architectural proteins HMGD1 and $\mathrm{H} 1$ bind reciprocally and have opposite effects on chromatin structure and gene regulation. BMC Genomics 2014; 15: 92.

73 Barski A, Zhao K. Genomic location analysis by ChIP-Seq. $J$ Cell Biochem 2009; 107: 11-18.

74 Brogaard K, Xi L, Wang JP, Widom J. A map of nucleosome positions in yeast at base-pair resolution. Nature 2012; 486: 496-501.

75 Brogaard KR, Xi L, Wang JP, Widom J. A chemical approach to mapping nucleosomes at base pair resolution in yeast. Methods Enzymol 2012; 513: 315-334.

76 Feng J, Liu T, Qin B, Zhang Y, Liu XS. Identifying ChIP-seq enrichment using MACS. Nat Protoc 2012; 7: 1728-1740.

77 Zhang Y, Liu T, Meyer CA et al. Model-based analysis of ChIP-Seq (MACS). Genome Biol 2008; 9: R137.

78 Heinz S, Benner C, Spann N et al. Simple combinations of lineage-determining transcription factors prime cis-regulatory elements required for macrophage and B cell identities. Mol Cell 2010; 38: 576-589.

79 Convertini P, Shen M, Potter PM et al. Sudemycin E influences alternative splicing and changes chromatin modifications. Nucleic Acids Res 2014; 42: 4947-4961.

80 Baas MC, Struijk GH, Moes DJ et al. Interstitial pneumonitis caused by everolimus: a case-cohort study in renal transplant recipients. Transplant Int 2014; 27: 428-436.

81 Kim D, Pertea G, Trapnell C, Pimentel H, Kelley R, Salzberg SL. TopHat2: accurate alignment of transcriptomes in the presence of insertions, deletions and gene fusions. Genome Biol 2013; 14: R36.

82 Zhu B, Fukada K, Zhu H, Kyprianou N. Prohibitin and cofilin are intracellular effectors of transforming growth factor beta signaling in human prostate cancer cells. Cancer Res 2006; 66: 8640-8647.

83 Adelman K, Wei W, Ardehali MB et al. Drosophila Paf1 modulates chromatin structure at actively transcribed genes. Mol Cell Biol 2006; 26: 250-260.

(Supplementary information is linked to the online version of the paper on the Cell Discovery website.)

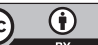

This work is licensed under a Creative Commons Attribution 4.0 International License. The images or other third party material in this article are included in the article's Creative Commons license, unless indicated otherwise in the credit line; if the material is not included under the Creative Commons license, users will need to obtain permission from the license holder to reproduce the material. To view a copy of this license, visit http://creativecommons.org/licenses/by/4.0/ 